\title{
GX 3+1: THE STABILITY OF SPECTRAL INDEX AS A FUNCTION OF MASS ACCRETION RATE
}

\author{
ELENA SEIFINA AND LeV TITARCHUK $2,3,4$ \\ ${ }^{1}$ Moscow State University/Stemberg Astronomical Institute, Universitetsky Prospect 13, Moscow 119992, Russia; seif@saimsu.ru \\ ${ }^{2}$ Dipartimento di Fisica, Università di Ferrara, Via Saragat 1, 1-44100 Ferrara, Italy; trarchnk@ fe.nfm.t \\ ${ }^{3}$ George Mason University, Fairfax, VA 22030, USA \\ ${ }^{4}$ Goddard Space Flight Center, NASA, Code 663, Greenbelt, MD 20770, USA; lev @ milkyway.gstc.nas \\ Received 2011 August 10; accepted 2011 December 27; published 2012 ???
}

\begin{abstract}
We present an analysis of the spectral and timing properties observed in X-rays from neutron star (NS) binary GX $3+1$ (4U 1744-26) during long-term transitions between the faint and bright phases superimposed on short-term transitions between lower banana (LB) and upper banana (UB) branches in terms of its color-color diagram. We analyze all observations of this source obtained with the Rossi X-ray Timing Explorer and BeppoSAX satellites. We find that the X-ray broadband energy spectra during these spectral transitions can be adequately reproduced by a composition of a low-temperature blackbody component, a Comptonized component (COMPTB), and Gaussian component. We argue that the electron temperature $k T_{e}$ of the Compton cloud monotonically increases from $2.3 \mathrm{keV}$ to $4.5 \mathrm{keV}$, when GX $3+1$ makes a transition from UB to LB. We also detect an evolution of noise components (a very low frequency noise and a high-frequency noise) during these LB-UB transitions. Using a disk seed photon normalization of $C O M P T B$, which is proportional to the mass accretion rate, we find that the photon power-law index $\Gamma$ is almost constant $(\Gamma=2.00 \pm 0.02)$ when mass accretion rate changes by factor four. In addition, we find that the emergent spectrum is dominated by the strong Comptonized component. We interpret this quasi-stability of the index $\Gamma$ and a particular form of the spectrum in the framework of a model in which the energy release in the transition layer located between the accretion disk and NS surface dominates that in the disk. Moreover, this index stability effect now established for GX $3+1$ was previously found in the atoll source 4U 1728-34 and suggested for a number of other low-mass X-ray NS binaries (see Farinelli \& Titarchuk). This intrinsic behavior of NSs, in particular for atoll sources, is fundamentally different from that seen in black hole binary sources where the index monotonically increases during spectral transition from the low state to the high state and then finally saturates at high values of mass accretion rate.
\end{abstract}

Key words: accretion, accretion disks - black hole physics - radiation mechanisms: non-thermal - stars: individual (GX 3+1) - stars: neutron

Online-only material: color figures

\section{INTRODUCTION}

Low mass X-ray binaries (LMXBs) hosting a neutron star (NS) show a variety of spectral states and transitions between them. In this regard, the so-called atoll sources (see, e.g., Hasinger \& van der Klis 1989; van der Klis 2005) are particularly interesting because they demonstrate a wide range of luminosities (the most of them show from 0.01 to 0.5 of the Eddington limit $L_{\text {Edd }}$ ). It is worth noting that the name of atoll sources is associated with the shape traced in the color-color diagram (CD). This shape can be divided into two main regions, corresponding to two X-ray states of the source: the harder one is related to the island state (IS) and the softer one is related to the banana (B) state.

These spectra of NS sources can be described by blackbody (BB) components, with color temperatures $k T_{\mathrm{BB}}<1 \mathrm{keV}$ and $k T_{s}>1 \mathrm{keV}$ that are presumably related to the accretion disk and NS surface, respectively. In addition, there is a thermal Comptonization component with electron temperature $k T_{e}=$ $2.3-15 \mathrm{keV}$ that is probably related to the transition layer (TL) located between the disk and NS (see Paizis et al. 2006; Farinelli \& Titarchuk 201 1; Seifina \& Titarchuk 2011, hereafter FT11 and ST11, respectively). An analysis of X-ray power spectra of atoll sources indicates to a tight relation between timing properties and the position on the CD, suggesting that the source timing and spectral properties are well determined by basic parameter such as mass accretion rate (see, e.g., Di Salvo et al. 2001).
GX $3+1$ is one of the brightest atoll sources associated with a bulge component of our Galaxy. GX $3+1$ along with GX 9+9, GX $9+1$, and GX $13+1$ form the subclass of persistently bright atoll sources, which are always in the banana state (see Hasinger $\&$ van der Klis 1989). In particular, two-branch structures have been observed in the CD and hardness-intensity diagram (HID) of GX 3+1 (Stella et al. 1985; Lewin et al. 1987; Schultz et al. 1989; Homan et al. 1998; Muno et al. 2002; Schnerr et al. 2003). Specifically, their tracks in the X-ray CD are long, diagonal, and slightly curved, while their fast timing properties are dominated only by a relatively weak ( $1 \%-4 \% \mathrm{rms}$ ) power-law shaped noise component. These aforementioned atoll sources are intermediate in terms of luminosity that changes in the range $0.1-0.5$ of $L_{\text {Edd }}$ (see Christian \& Swank 1997 ; Ford \& van der Klis 2000 ).

In contrast to other atoll sources and Z sources, these bright atoll sources have so far not shown $\mathrm{kHz}$ QPOs (see Wijnands et al. 1998; Strohmayer 1998; Homan et al. 1998; Oosterbroek et al. 2001; Schnerr et al. 2003), which can be attributed to the fact that these objects do not reach the left lower banana (LLB), where other atoll sources exhibit $\mathrm{kHz}$ QPOs (van der Klis 2000). For example, the weaker atoll sources, such as, e.g., $4 \mathrm{U} 1608-52,4 \mathrm{U} 0614+09$, or $4 \mathrm{U} 1728-34$, show $\mathrm{kHz}$ QPOs in LLB.

X-ray data of GX $3+1$ ( 4 U 1744-26) obtained in broad energy ranges using BeppoSAX $(0.1-200 \mathrm{keV})$ and Rossi $X$-Ray Timing Explorer (RXTE) $(3-200 \mathrm{keV})$ offer a unique 
possibility to further investigate, in detail, the evolution of $\mathrm{X}$-ray spectral properties during transition events. This bright atoll source shows long-term transitions from the fainter phase to its brighter phase in X-rays and vice versa when the corresponding luminosity changes, at least, by a factor of four, while on timescales of hours $\mathrm{GX} 3+1$ demonstrates low flux variabilities as transitions between LB and upper banana (UB) states. Naturally one can pose a fair question: what is the physical mechanism responsible for the source emission during these luminosity changes and particularly how the spectral index changes during these transitions?

GX 3+1 was discovered during an Aerobee-rocket flight on 1964 June 16 (Bowyer et al. 1965). Subsequently, this source was observed many times during various observational campaigns. Detailed long-term monitoring observations of GX $3+1$ were performed by all-sky monitor (ASM) on GINGA (see Asai et al. 1993), EXOSAT (see Schultz et al. 1989), RXTE (see Bradt et al. 1993; Kuulkers \& van der Klis 2000), Wide-Field Camera (WFC) of BeppoSAX (see den Hartog et al. 2003), and also by INTEGRAL (see Paizis et al. 2006). In particular, den Hartog et al. (2003) found three types of variability: short-term variation (of order of seconds), mild variability on a daily (hourly) timescale, and slow sinusoidal-like variation on a timescale of years. However, it is surprising that the hardness ratio, which can be a measure of the spectral shape, stays almost constant during these observations.

Although an optical counterpart has not yet been identified (e.g., Naylor et al. 1991) GX 3+1 is presumably a low-mass $\mathrm{X}$-ray binary in which a NS is accompanied by a low-mass star of spectral type $A$ or a later. During an active stage the companion overflows its Roche lobe and transfers matter onto the NS via an accretion disk. This process is possibly accompanied by nuclear burning of helium or hydrogen layer of NS surface as a result of the matter accumulation on the NS surface (Hanson \& van Horn 1975). Unstable fusion occurs leading to thermonuclear flashes which can be observable in the form of $X$-ray bursts, so-called type-I X-ray bursts (Woosley \& Taam 1976). GX 3+1 does exhibit fast variability in the form of type-I X-ray bursts, which were extensively studied by a number of X-ray missions, GINGA (Asai et al. 1993), Granat (Pavlinsky et al. 1994; Molkov et al. 1999), RXTE (Kuulkers \& van der Klis 2000; Kuulkers 2002), and by INTEGRAL (Chenevez et al. 2006).

A unique super-burst with a decay time of $1.6 \mathrm{hr}$ was detected with the ASM on RXTE (Kuulkers 2002). One of the shortest bursts ever seen exhibits a quick (i.e., less than 2 s) radius expansion phase, indicating that the burst luminosity was at the Eddington luminosity, causing the NS atmosphere to expand due to radiation pressure. This implies that a distance to the source is about $4.5 \mathrm{kpc}$, assuming that the NS atmosphere is hydrogen-rich (see more details in Kuulkers \& van der Klis $2000)$.

In this paper, we concentrate our efforts on the spectral and timing properties of $\mathrm{GX} 3+1$ related to changes in mass accretion rate, which are seen as the mild and slow variabilities. Previously, an analysis of the burst properties of GX $3+1$ as a function of mass accretion rate on the timescales larger than one minute was presented by Asai et al. (1993) and den Hartog et al. (2003). In particular, the slow variability has been revealed during transitions from the faint phase to the bright phase of luminosity and is generally caused by significant increase of mass accretion rate. The mild variability is presumably related to moderate local variability of mass accretion rate and revealed as local transitions between $L B$ and $U B$ states. The slow variability has been investigated with the RXTE/ASM light curve, WFC of BeppoSAX (den Hartog et al. 2003), and the ASM on GINGA (Asai et al. 1993) and these observations indicate that the flux oscillates semi-sinusoidally with a period of 6-7 years (see Figure 1).

Moreover, the GINGA measurements with its Large Area Detector (Asai et al. 1993) show a constant 1-20 keV spectral shape over the fainter phases and also during brighter phases on luminosity suggest a constancy of the spectral index.

This stability of the $\mathrm{X}$-ray spectral shape over the bright and faint phases was also been confirmed by the WFC BeppoSAX measurements in the $2-28 \mathrm{keV}$ band which showed minimal spectral changes (den Hartog et al. 2003).

The stability of the index was noted previously by FT11, for a number of NS LMXB at different luminosities. They collected $X$-ray spectra obtained by BeppoSAX and demonstrated the relative stability of spectral index $\alpha$ approximately of $1(\Gamma=$ $\alpha+1$ ) for quite a few NS sources X 1658-298, GX 354-0, GS 1826-238, 1E 1724-3045, Cyg X-1, Sco X-1, GX 17+2, and GX $349+2$ at different spectral states. Recently ST11 presented results of analysis of X-ray spectra for "atoll" source 4U 172834, detected with BeppoSAX and RTXE at different luminosities and values of the best-fit electron temperatures. These results indicate that the value of the index varies slightly about a value of 1 (or the photon index varies around 2) independently of the electron temperature of the Compton cloud (CC) $k T_{e}$ and luminosity. This unique stability of the photon index may be an intrinsic property of NS at least for the atoll sources. It is probably determined by common physical conditions for this class of sources. FT11 and ST11 interpreted this quasi-stability of the index $\Gamma$ in the framework of a model in which the spectrum is dominated by a strong thermal Comptonized component formed in the TL located between the accretion disk and NS surface. Indeed, the index quasi-stability takes place when the energy release in the TL is much higher than the photon energy flux coming from the accretion disk and illuminating the TL.

The evolution of spectral parameters of compact objects in $\mathrm{X}$-ray binaries is of great interest for understanding their nature. It is well known that many black hole $(\mathrm{BH})$ candidate binaries exhibit correlations between mass accretion rate $\dot{M}$ and photon power-law index $\Gamma$ (see Shaposhnikov \& Titarchuk 2009 and Titarchuk \& Seifina 2009, hereafter ST09 and TS09, respectively). In the soft states of BHs these index- $\dot{M}$ correlations almost in any case show a saturation of $\Gamma$ at high values of the $\dot{M}$. This saturation effect can be considered as a BH signature or equivalently as a signature of a converging flow into BH (ST09 and TS09).

In this paper, we present the analysis of the BeppoSAX available observations and $R X T E / P C A$ observations during 1996-2010 years for GX 3+1. In Section 2, we present the list of observations used in our data analysis while in Section 3 , we provide the details of $X$-ray spectral analysis. We analyze an evolution of $X$-ray spectral and timing properties during the state transition in Section 4. We make our conclusions in Section 5.

\section{DATA SELECTION}

Broadband energy spectra of the source were obtained combining data from three BeppoSAX Narrow Field Instruments (NFIs): the Low Energy Concentrator Spectrometer (LECS) for $0.3-4 \mathrm{keV}$ (Parmar et al. 1997), the Medium Energy Concentrator Spectrometer (MECS) for $1.8-10 \mathrm{keV}$ (Boella et al. 1997 ), and the Phoswich Detection System (PDS) for $15-60 \mathrm{keV}$ 


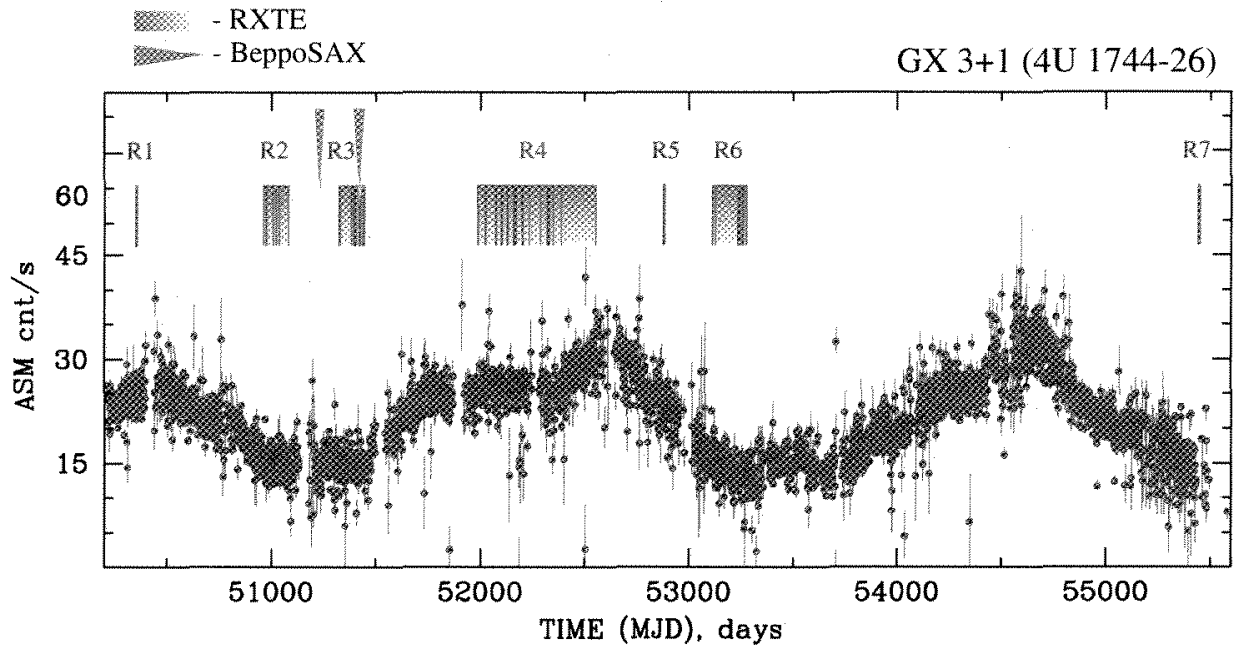

Figure 1. Evolution of ASM/RXTE count rate during 1996-2010 observations of GX $3+1$. Blue vertical strips (on the top of the panel) indicate to time for the RXTE pointed observations. Whereas bright blue rectangles are related to the RXTE data sets listed in Table 1, green triangles show BeppoSAX NFI data listed in Table 2 . (A color version of this figure is available in the online journal.)

Table 1

The List of BeppoSAX Observations of GX $3+1$ Used in Our Analysis

\begin{tabular}{lcccc}
\hline \hline Obs. ID & Start Time (UT) & End Time (UT) & MJD Interval & CD State \\
\hline 20603001 & 1999 Feb 28 11:02:15 & 1999 Feb. 30 09:14:15 & $51237.4-51238.9$ & Upper banana \\
20835001 & 1999 Aug 30 18:33:08 & 1999 Aug. 31 04:54:32 & $51420.8-51421.9^{1}$ & Lower banana \\
\hline
\end{tabular}

Reference. (1) Oosterbroek et al. 2001.

(Frontera et al. 1997). The SAXDAS data analysis package is used for processing data. For each of the instruments we performed the spectral analysis in the energy range for which response matrix is well determined. The LECS data have been re-normalized based on MECS. Relative normalization of the NFIs was treated as free parameters in model fitting, except for the MECS normalization that was fixed at a value of 1 . We then checked this fitting procedure if these normalizations were in a standard range for each instruments. ${ }^{5}$ In addition, spectra are rebinned accordingly to energy resolution of the instruments in order to obtain significant data points. We rebinned the LECS spectra with a binning factor which is not constant over energy (Section 3.1.6 of Cookbook for the BeppoSAX NFI spectral analysis) using template files in GRPPHA of XSPEC. Also we rebinned the PDS spectra with a linear binning factor of two, grouping two bins together (resulting bin width is $1 \mathrm{keV}$ ). Systematic error of $1 \%$ has been applied to these analyzed spectra. In Table 1, we listed the BeppoSAX observations used in our analysis.

We have analyzed the available data obtained with $R X T E$ (Bradt et al. 1993) which have been found in the time period from 1996 October to 2010 September (see also a review by Galloway et al. 2008). In our investigation we selected 101 observations made at different count rates (luminosity states) with a good coverage of rise-decay transition tracks. We have made an analysis of RXTE observations of GX $3+1$ during 14 years for seven intervals indicated by blue rectangles in Figure I (top).

RXTE/PCA spectra have been extracted and analyzed, wherein PCA Standard 2 mode data, collected in the $3-50 \mathrm{keV}$

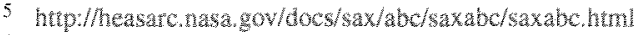

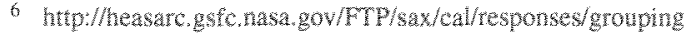

energy range, using the most recent release of PCA response calibration (ftool pcarmf v11.1). The relevant dead-time corrections to energy spectra have been applied. We used the data which are available through the GSFC public archive (http:/heasarc.gsfc.nasa.gov). In Table 2, we presented the groups of RXTE observations which cover the source evolution from faint to bright (phase) events. Note that available $R X T E$ data contain one "bright phase" set $(R 4)$ and six "faint phase" set $(R 1-R 3, R 5-R 7)$. The PCA energy spectra were modeled using XSPEC astrophysical fitting software. Systematic error of $0.5 \%$ has been applied to the analyzed spectra.

We have also used public GX $3+1$ data from the ASM on board $R X T E$, which show long-term quasi-periodic variability of the mean soft flux during $\sim$ six year cycle (Figure 1). We use definitions of the fainter and brighter on luminosity phases to relate these phases to the source luminosity and we demonstrate that during the bright/faint phase transition of GX 3+1 COMPTB normalization changes from 0.04 to 0.14 $L_{39}^{\text {soft }} / D^{2}{ }_{10}$ where $L_{39}^{\text {soft }}$ is the soft photon luminosity in units of $10^{39} \mathrm{erg} \mathrm{s}^{-1}$ and $D_{10}$ in units of $10 \mathrm{kpc}$ is distance to the source.

\section{SPECTRAL ANALYSIS}

In our spectral data analysis, we use a model which consists a sum of a Comptonization component (COMPTB) (COMPTB is an XSPEC contributed model, see Farinelli et al. 2008, hereafter F08), soft blackbody component of temperature $T_{\mathrm{BB}}$, and Gaussian line component. The COMPTB spectral component has the following parameters: temperature of the seed photons $T_{s}$, energy index of the Comptonization spectrum $\alpha(=\Gamma-1)$, electron temperature $T_{e}$, illumination (Comptonization) fraction

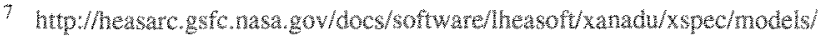
comptobuth
} 


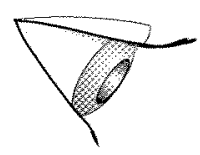

Black-body like emission of $\mathrm{NS}\left(\mathrm{kT} \mathrm{T}_{\mathrm{s}}>1 \mathrm{keV}\right)$

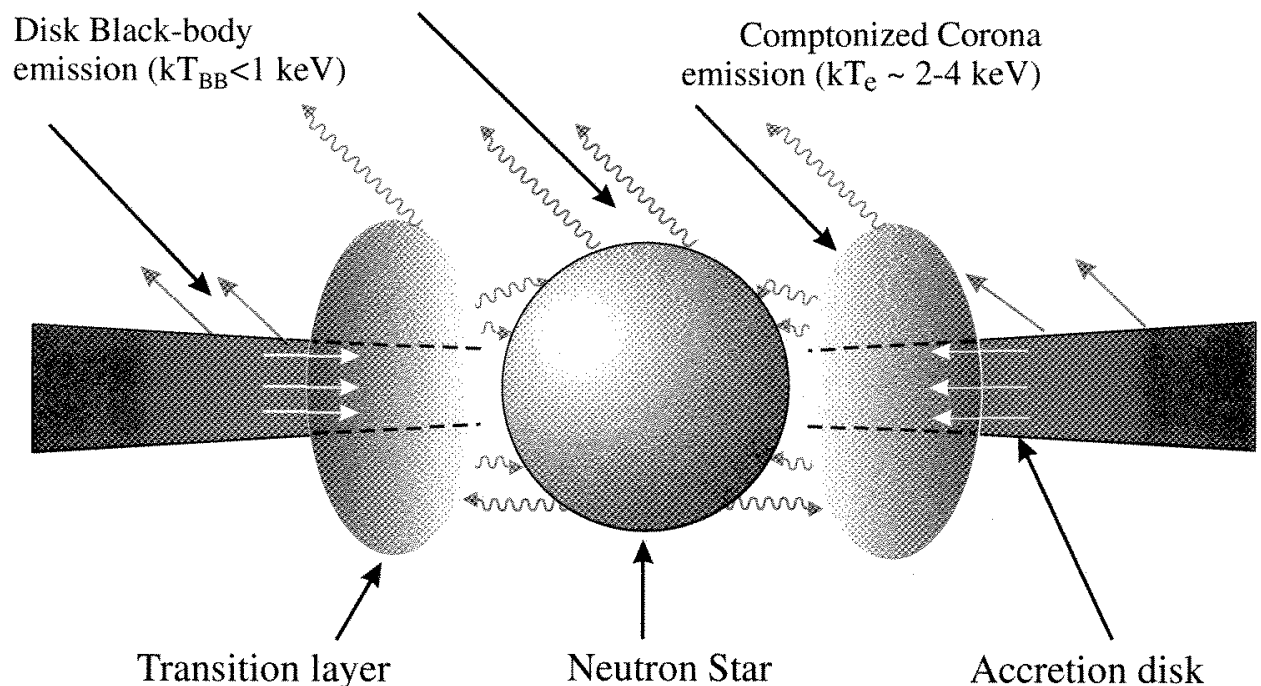

Figure 2. Suggested geometry of GX $3+1$. Disk and neutron star soft photons are upscattered off hotter plasma of the transition layer located between the accretion disk and NS surface. Some fraction of these photons is seen directly by the Earth observer. Red and blue photon trajectories correspond to soft and hard (upscattered) photons, respectively.

(A color version of this figure is available in the online joumal.)

Table 2

The List of RXTE Observation Groups of GX $3+1$

\begin{tabular}{llclc}
\hline \hline Number of Set & Dates, MJD & RXTE Proposal ID & Dates UT & $\begin{array}{c}\text { Phase on } \\
\text { Lumonosity }\end{array}$ \\
\hline R1 & 50365 & 10069 & 1996 Oct 903:36:00-04:08:00 & faint \\
R2 & $50962-51118$ & 30042,30048 & 1998 May 29-Nov 1 & faint \\
R3 & $51324-51445$ & $40023,40425^{1}$ & 1999 May 26-Sept 24 & faint \\
R4 & $51998-52554$ & 60022 & 2001 March 30-2002 Oct 7 & bright \\
R5 & 52881 & 80105 & 2003 Aug 30 03:53:36-06:29:13 & faint \\
R6 & $53108-53280$ & 90022 & 2004 Apr 13-Oct 2 & faint \\
R7 & $55440.6-55440.8$ & 94307 & 2010 Sept 1 15:00:32-21:07:58 & faint \\
\hline
\end{tabular}

Reference. (1) Oosterbroek et al. 2001.

$f$ of the CC by the soft (NS) photons, $f=A /(1+A)$, and the normalization of the seed (NS) photon spectrum $N_{\mathrm{COM}}$.

We include a simple Gaussian component in the model, whose parameters are a centroid line energy $E_{\text {line }}$, the width of the line $\sigma_{\text {line }}$, and the normalization $N_{\text {line }}$ to fit the data in the $6-8 \mathrm{keV}$ energy range. We also use the interstellar absorption with a column density $N_{\mathrm{H}}$. It should be noted that we fixed certain parameters of the COMPTB component: $\gamma=3$ (low energy index of the seed photon spectrum) and $\delta=0$ because we neglect an efficiency of the bulk inflow effect versus the thermal Comptonization for NS GX $3+1$. We apply a value of hydrogen column $N_{\mathrm{H}}=1.6 \times 10^{22} \mathrm{~cm}^{-2}$, which was found by Oosterbroek et al. (2001).

Initially, we have tried a model consisting of an absorbed thermal component (bbody) and a thermal Comptonization component (COMPTB) but this model gave a poor description of data. Significant positive residuals around $\sim 6.5 \mathrm{keV}$ suggest the presence of fluorescent iron emission line. The addition of
Gaussian line component at $6.4 \mathrm{keV}$ considerably improves fit quality and provides a statistically acceptable $\chi_{\text {red }}^{2}$.

At the first time the fluorescent iron emission line in GX $3+1$ was detected by Oosterbroek et al. (2001) using BeppoSAX on 1999 August 30 (id=20835001). Oosterbroek et al. (2001) successfully described this emission feature with the Gaussian line model and they used a model consisting of thermal component (dominating energy release around $1 \mathrm{keV}$ ) and a thermal Comptonization tail to describe the $0.1-50 \mathrm{keV}$ continuum. However, they needed to add a $2 \%$ systematical uncertainty to LECS and MECS data to obtain acceptable $\chi_{\text {red }}^{2}$. We investigate a possibility to further improve a quality of the fit.

In Figure 2, we illustrate our spectral model as a basic model for fitting the BeppoSAX and RXTE spectral data for GX $3+1$. We assume that accretion onto a NS takes place when the material passing through the two main regions: a geometrically thin accretion disk (the standard Shakura-Sunyaev disk, see Shakura 
Table 3

Best-fit Patameters of Spectral Analysis of BeppoSAX Observations of GX $3+1$ in $0.3-50$ keV Energy Range

\begin{tabular}{|c|c|c|c|c|c|c|c|c|c|c|c|}
\hline $\begin{array}{l}\text { Observational } \\
\text { ID }\end{array}$ & $\begin{array}{l}\text { MuD, } \\
\text { (day) }\end{array}$ & $\begin{array}{c}T_{\mathrm{BB}} \\
(\mathrm{keV})\end{array}$ & $N_{\mathrm{BB}}{ }^{b}$ & $\begin{array}{c}T_{S} \\
(\mathrm{keV})\end{array}$ & $\begin{array}{c}\alpha= \\
\Gamma-1\end{array}$ & $\begin{array}{c}T_{e} \\
(\mathrm{keV})\end{array}$ & $\log (A)$ & $N_{\mathrm{COM}^{b}}$ & $\begin{array}{c}E_{\text {line }} \\
(\mathrm{keV})\end{array}$ & $N_{\text {line }}{ }^{b}$ & $\overline{\chi_{\text {red }}^{2}(\text { dof })}$ \\
\hline 20603001 & 51237.5 & $0.47(3)$ & $2.65(2)$ & $1.30(3)$ & $0.99(7)$ & $3.68(5)$ & $0.09(4)$ & $4.18(3)$ & $7.4(1)$ & $0.55(4)$ & $1.08(457)$ \\
\hline 20835001 & 51420.8 & $0.62(5)$ & $1.61(1)$ & $1.21(5)$ & $1.07(4)$ & $2.4(2)$ & $-0.32(8)$ & $3.56(2)$ & $6.0(1)$ & $0.43(4)$ & $1.16(445)$ \\
\hline
\end{tabular}

Notes. Parameter errors correspond to lo confidence level

a The spectral model is wabs * (blackbody+COMPTB + Gaussian), normalization parameters of blackbody and COMPTB components are in units of $L_{37}^{\text {soft } / d_{10}^{2}}$ where $L_{37}^{\text {sofi }}$ is the soft photon luminosity in units of $10^{37} \mathrm{erg} \mathrm{s}^{-1}, d_{10}$ is the distance to the source in units of $10 \mathrm{kpc}$, and Gaussian component is in units of $10^{-2} \times$ total photons $\mathrm{cm}^{-2} \mathrm{~s}^{-1}$ in line.

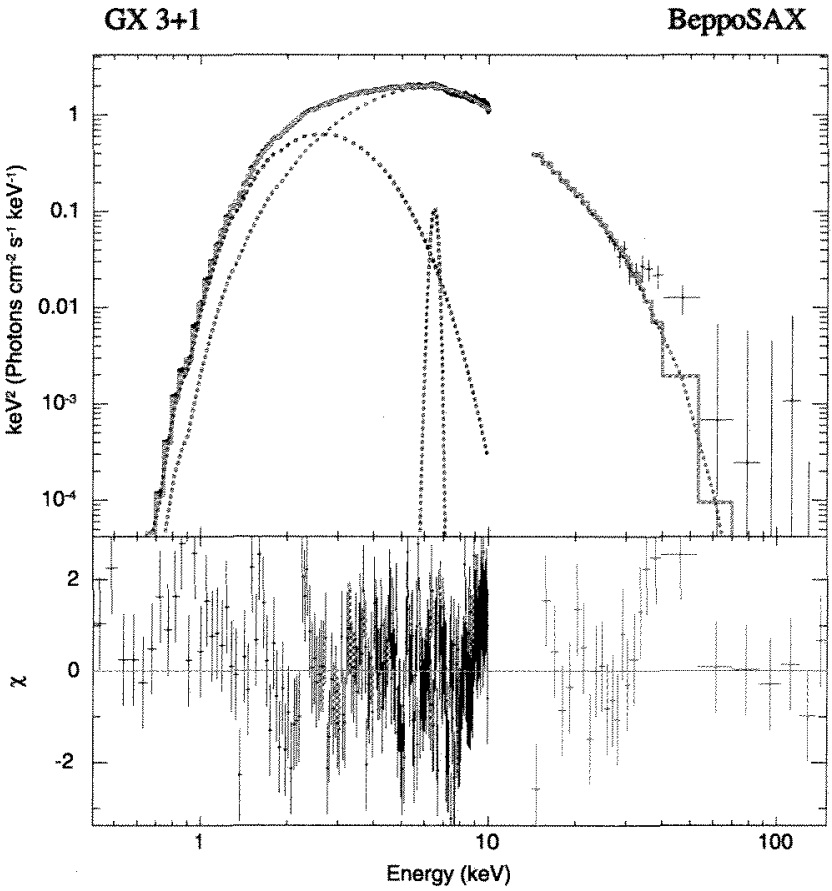

Figure 3. Top: the best-fit spectrum of $\mathrm{GX} 3+1$ in $E * F(E)$ units using BeppoSAX observation 20603001 carried out on 1999 February 28 -March 1 The data are presented by crosses and the best-fit spectral model wabs (blackbody+COMPTB+Gaussian) by green line. The model components are shown by blue, red, and crimson lines for blackbdody, COMPTB, and Gaussian components, respectively. Bottom: $\Delta \chi \mathrm{vs}$. photon energy in keV. The best-fit model parameters are $\Gamma=1.99 \pm 0.07, T_{e}=3.68 \pm 0.05 \mathrm{keV}$, and $E_{\text {line }}=$ $7.4 \pm 0.1 \mathrm{keV}$ (reduced $\chi^{2}=1.08$ for 457 dof, see more details in Table 3 ).

(A color version of this figure is available in the online joumal.)

\& Sunyaev 1973) and the TL, where NS and disk soft photons are upscattered off hot electrons. In other words, in our picture, the emergent thermal Comptonization spectrum is formed in the TL, where thermal disk seed photons and soft photons from the NS are upscattered off the relatively hot plasma (electrons). Some fraction of these seed soft photons can be also seen directly. Red and blue photon trajectories shown in Figure 2 correspond to soft (seed) and hard (upscattered) photons, respectively.

We show examples of X-ray spectra in Figures 3 and 4 for BeppoSAX and RXTE data, respectively. Spectral analysis of BeppoSAX and RXTE observations indicates that $X$-ray spectra of $G X 3+1$ can be described by a model with a Comptonization component represented by the COMPTB model. Moreover, for broadband BeppoSAX observations this spectral model component is modified by photoelectric absorption at low energies.

On the top of Figure 3 we demonstrate the best-fit BeppoSAX spectrum of GX $3+1$ in units of $E * F(E)$ (top) (where $F(E)$

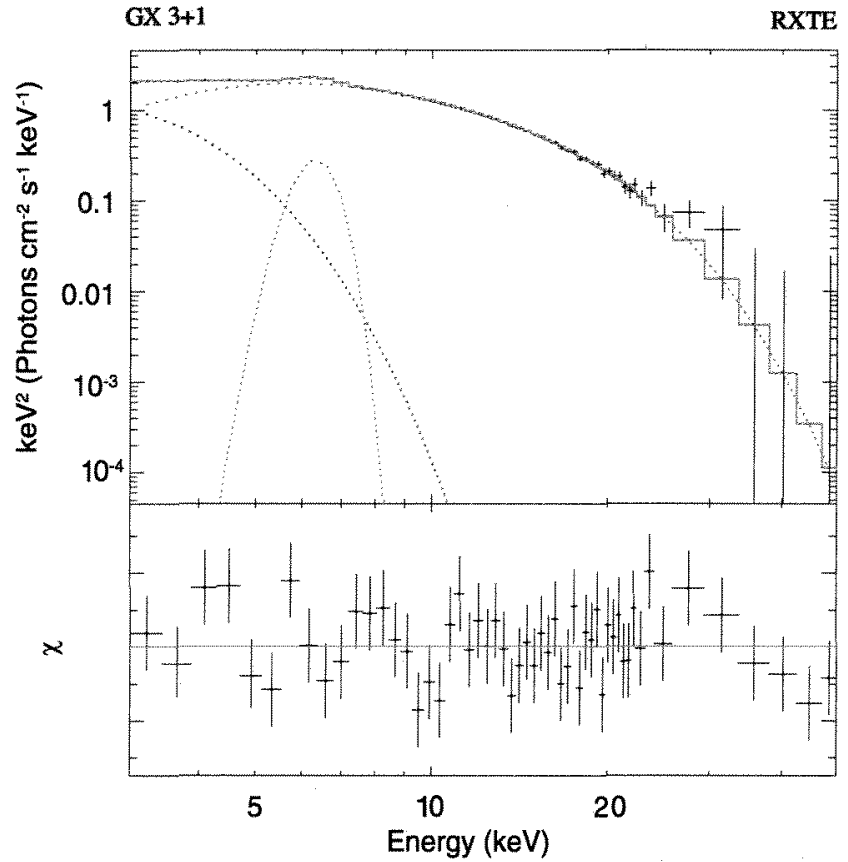

Figure 4. Upper panel: a typical $E * F(E)$ spectral diagram of $\mathrm{GX} 3+1$ for PCA/RXTE observation 94307-05-01-000 on 2010 September 1 (MJD 55431.62) during the faint phase (green line). The spectral model, presented by its components, is shown by dashed red, blue, and purple lines for COMPTB, blackbody, and Gaussian components, respectively. Bottom panel: $\Delta \chi$ vs. photon energy in keV. The best-fit model parameters are $\Gamma=2.04 \pm 0.04$, $k T_{e}=3.16 \pm 0.05 \mathrm{keV}$, and $E_{\text {line }}=6.59 \pm 0.05 \mathrm{keV}$ (reduced $\chi^{2}=0.87$ for 67 dof, see more details in Table 4 ).

(A color version of this figure is available in the online journal.)

is energy flux in erg, per keV and per second) using our model for BeppoSAX observation (id=20603001) carried out on 1999 February 28-March 1. The data are presented by crosses and the best-fit spectral model wabs*blackbody + COMPTB + Gaussian) by green line. The model components are shown by blue, red, and crimson lines for blackbdody, COMPTB, and Gaussian components, respectively. On the bottom we show $\Delta \chi$ versus photon energy in $\mathrm{keV}$. The best-fit model parameters are $\Gamma=1.99 \pm 0.07, k T_{e}=3.68 \pm 0.05 \mathrm{keV}$, and $E_{\text {line }}=7.4 \pm$ $0.1 \mathrm{keV}$ (reduced $\chi^{2}=1.08$ for 457 dof; see more details in Table 3 ). In particular, we find that an addition of the soft thermal component with temperature $k T_{\mathrm{BB}}=0.5-0.7 \mathrm{keV}$ to the model significantly improves the fit quality of the BeppoSAX spectra. For the BeppoSAX data (see Tables 1 and 3) we find that the spectral index $\alpha$ is of $1.03 \pm 0.04$ (or the corresponding photon index $\Gamma=\alpha+1$ is $2.03 \pm 0.04$ ).

Unfortunately RXTE detectors do not provide well-calibrated spectra below $3 \mathrm{keV}$ while the broad energy band of BeppoSAX 
Table 4

The Best-fit Parameters of Spectral Analysis of PCA/RXTE Observations of GX $3+1$ in 3-50 keV Energy Range

\begin{tabular}{|c|c|c|c|c|c|c|c|c|c|c|c|c|}
\hline $\begin{array}{l}\text { Observational } \\
\text { ID }\end{array}$ & $\begin{array}{l}\text { MJD, } \\
\text { (day) }\end{array}$ & $\begin{array}{c}\alpha= \\
\Gamma-1\end{array}$ & $\begin{array}{c}T_{e} \\
(\mathrm{keV})\end{array}$ & $\log (A)$ & $N_{\mathrm{COM}^{b}}$ & $\begin{array}{c}T_{s}, \\
(\mathrm{keV})\end{array}$ & $N_{\text {Bbody }}{ }^{b}$ & $\begin{array}{c}E_{\text {line }}, \\
(\mathrm{keV})\end{array}$ & $\begin{array}{l}\text { Oline, } \\
(\mathrm{keV})\end{array}$ & $N_{\text {line }}{ }^{b}$ & $x_{\text {red }}^{2}($ dof $)$ & $\overline{F_{1} / F_{2} c}$ \\
\hline $10069-03-01-00$ & 50365.172 & $1.00(8)$ & $2.40(2)$ & $0.03(1)$ & $10.88(2)$ & $1.10(8)$ & $2.74(5)$ & $6.53(3)$ & $0.58(5)$ & $0.95(4)$ & $1.1(67)$ & $6.79 / 1.28$ \\
\hline $30042-04-01-00$ & 50962.598 & $1.0(1)$ & $2.52(1)$ & $-0.04(3)$ & $6.3(1)$ & $1.65(5)$ & $3.17(3)$ & $6.51(2)$ & $0.5(1)$ & $0.46(2)$ & $1.50(67)$ & $4.13 / 0.80$ \\
\hline $30042-04-02-00$ & 50973.668 & $1.02(7)$ & $2.42(1)$ & $-0.1(1)$ & $9.9(1)$ & $1.17(4)$ & $2.6(1)$ & $6.5(1)$ & $0.5(1)$ & $0.37(8)$ & $1.12(67)$ & $2.69 / 1.49$ \\
\hline $30048-01-01-00$ & 51011.138 & $0.99(2)$ & $2.44(2)$ & $0.18(8)$ & $6.3(2)$ & $1.45(8)$ & $3.12(8)$ & $6.42(8)$ & $0.6(1)$ & $0.7(1)$ & $0.82(67)$ & $4.68 / 1.03$ \\
\hline $30042-04 \sim 03-00$ & 51113.941 & $1.01(2)$ & $3.15(2)$ & $0.39(5)$ & $4.00(6)$ & $1.5(1)$ & $3.05(2)$ & $6.53(1)$ & $0.50(8)$ & $0.42(1)$ & $0.87(67)$ & $3.26 / 1.31$ \\
\hline $30042-04-03-01$ & 51114.207 & $1.03(3)$ & $3.17(2)$ & $0.41(5)$ & $4.07(6)$ & $1.5(1)$ & $3.07(2)$ & $6.58(1)$ & $0.52(7)$ & $0.44(1)$ & $0.91(67)$ & $3.62 / 1.56$ \\
\hline $30042-04-04-00$ & 51118.739 & $1.1(1)$ & $3.52(8)$ & $0.45(6)$ & $3.7(1)$ & $1.7(5)$ & $2.86(3)$ & $6.51(4)$ & $0.6(2)$ & $0.47(2)$ & & $3.03 / 1.34$ \\
\hline $40023-01-01-00$ & 51324.737 & $1.0(3)$ & $2.48(2)$ & $0.02(1)$ & $5.98(8)$ & $1.6(2)$ & $2.62(8)$ & $6.43(9)$ & $0.67(9)$ & $0.9(1)$ & $1.35(67)$ & $4.68 / 1.03$ \\
\hline $40023-01-01-01$ & 51325.310 & $1.0(2)$ & $2.40(1)$ & $0.23(3)$ & $5.78(6)$ & $1.6(2)$ & $2.64(9)$ & $6.43(5)$ & $0.67(8)$ & $0.73(7)$ & $0.75(67)$ & $4.15 / 0.88$ \\
\hline $40023-01-01-02$ & 51325.542 & $1 .(1)$ & $2.46(1)$ & $-0.01(1)$ & $5.85(4)$ & $1.6(2)$ & $2.38(8)$ & $6.45(5)$ & $0.62(6)$ & $0.73(8)$ & $1.26(67)$ & $4.06 / 0.77$ \\
\hline $40023-01-03-00$ & 51390.248 & $1.0(1)$ & $2.54(1)$ & $0.01(1)$ & $6.20(6)$ & $1.56(8)$ & $2.6(1)$ & $6.49(7)$ & $0.60(7)$ & $0.7(1)$ & $0.94(67)$ & $4.33 / 0.88$ \\
\hline $40023-01-02-00$ & 51398.243 & $0.9(2)$ & $2.53(1)$ & $0.04(2)$ & $5.61(5)$ & $1.4(1)$ & $2.68(9)$ & $6.45(5)$ & $0.64(6)$ & $0.76(9)$ & 1.01 & $3.99 / 0.81$ \\
\hline $40023-01-02-01$ & 51398.188 & $0.9(1)$ & $2.50(2)$ & $0.02(2)$ & $5.50(5)$ & $1.5(1)$ & $2.78(7)$ & $6.45(5)$ & $0.48(8)$ & $0.61(9)$ & 1.011 & $3.93 / 0.76$ \\
\hline $40023-01 \sim 02-02$ & 51399.644 & $0.9(1)$ & $2.49(8)$ & $0.01(1)$ & $6.93(8)$ & $1.2(1)$ & $2.47(5)$ & $6.46(9)$ & $0.69(9)$ & $0.96(8)$ & $0.53(67)$ & $4.74 /$ \\
\hline $40023-01-02-03$ & 51400.131 & $1.01(6)$ & $2.51(5)$ & $0.06(2)$ & $6.19(7)$ & $1.2(1)$ & $3.16(4)$ & & & $1.09(6)$ & 0.71 & $4.34 / 0.86$ \\
\hline $40023-01-02-04$ & 51400.192 & $1.01(9)$ & $2.4(1)$ & $0.06(2)$ & $5.77(6)$ & $1.18(3)$ & $2.83(5)$ & $6.45(9)$ & $0.52(5)$ & $0.61(6)$ & $0.85(67)$ & $3.97 / 0.75$ \\
\hline $40023-01-02-05$ & 51400.259 & $1.0(1)$ & $2.6(1)$ & $-0.09(5)$ & $6.72(6)$ & $1.25(2)$ & $2.9(1)$ & $6.50(7)$ & $0.46(7)$ & $0.63(9)$ & 0.77 & 4.64 \\
\hline $40023-01-02-06$ & 51400.325 & $1.0(1)$ & $2.45(7)$ & $0.18(9)$ & $6.19(5)$ & $1.25(8)$ & $3.04(8)$ & 6.4 & & & & 4.26 \\
\hline 40023-01-02-07 & 51400.723 & $1.0(1)$ & $2.45(1)$ & $0.11(2)$ & $6.85(4)$ & $1.20(8)$ & $2.38(7)$ & 6.4 & $0.47(5)$ & $0.45(8)$ & 0.92 & $4.70 /$ \\
\hline $40023-01-04-00$ & 51404.304 & $0.99(1)$ & $4.50(4)$ & $-0.9(1)$ & $3.99(9)$ & $1.82(3)$ & $3.63(9)$ & $4.55(5)$ & $0.50(7)$ & $0.70(6)$ & $1.70(67)$ & $4.16 / 0.94$ \\
\hline $40023-01-03-01$ & 51405.307 & $1 .(1)$ & $2.48(1)$ & $0.09(2)$ & $6.38(6)$ & $1.20(8)$ & $2.32(9)$ & $6.55(8)$ & & 0.6 & & $4.39 /$ \\
\hline $40425-01-01-00$ & 51420.779 & $1.0(1)$ & $3.05(7)$ & $-0.5(5)$ & $6.05(8)$ & $1.59(8)$ & $4.01(6)$ & $6.5(1)$ & $0.6(1)$ & 1.0 & 0.82 & $4.84 / 0.97$ \\
\hline $40425-01-01-01$ & 51420.846 & $1.09(5)$ & $2.83(9)$ & $-0.31(7)$ & $6.85(7)$ & $1.59(9)$ & $4.09(9)$ & $6.5(1)$ & $0.62(9)$ & $0.87(9)$ & 1.05 & $5.36 / 1.21$ \\
\hline $40425-01-01-02$ & 51421.018 & $1.05(1)$ & $2.8(1)$ & $-0.29(3)$ & $6.23(2)$ & $1.59(5)$ & $3.74(9)$ & $6.5(1)$ & 0.6 & & & $4.88 / 1.10$ \\
\hline $40425-01-01-03$ & 51421.084 & $1.01(1)$ & $3.29(9)$ & $-0.29(3)$ & $5.83(5)$ & $1.59(8)$ & $4.19(6)$ & $6.5(1)$ & 0.65 & 0.86 & 1.3 & $4.87 / 0.98$ \\
\hline $40425-01-01-04$ & 51421.151 & $1.0(1)$ & $2.85(8)$ & $-0.33(6)$ & $4.77(4)$ & $1.55(7)$ & $3.91(3)$ & $6.38(2)$ & $0.58(7)$ & $0.99(2)$ & 1.15 & $4.87 / 0.98$ \\
\hline $40425-01-01-05$ & 51421.218 & $1.08(9)$ & $2.99(5)$ & $-0.43(3)$ & $5.22(5)$ & $1.49(8)$ & $4.11(2)$ & $6.38(4)$ & $0.59(4)$ & $1.11(8)$ & 1.39 & $4.47 / 0.88$ \\
\hline $40425 \cdots 01-01-06$ & 51421.285 & $1.02(8)$ & $2.69(6)$ & $-0.19(6)$ & $4.24(2)$ & $1.35(7)$ & $3.79(3)$ & $6.38(3)$ & $0.62(8)$ & $0.87(6)$ & 1.36 & $3.77 / 0.74$ \\
\hline $40023-01-05-00$ & 51445.320 & $0.99(3)$ & $2.59(9)$ & $-0.06(4)$ & & $1.24(8)$ & $2.7(1)$ & $6.47(8)$ & $0.46(4)$ & $0.62(4)$ & 0.84 & $4.96 / 1.03$ \\
\hline $40023-01-03-01$ & 51445.599 & $1.0(1)$ & $2.35(4)$ & $0.73(9)$ & $6.44(7)$ & $1.16(7)$ & $2.32(1)$ & $6.54(9)$ & $0.4(1)$ & $0.52(8)$ & 1.7 & $4.80 / 1.13$ \\
\hline $60022-01-01-00$ & 51998.64 & $1.06(3)$ & $2.49(1)$ & $0.04(1)$ & $12.03(1)$ & $1.20(5)$ & $2.86(2)$ & $6.24(2)$ & $0.5(1$ & $1.50(6)$ & $1.07(67)$ & $7.94 / 1.69$ \\
\hline $60022-01-01-01$ & 51998.71 & $1.03(9)$ & $2.52(2)$ & $0.07(4)$ & & $1.20(7)$ & $2.31(3)$ & $6.5(1)$ & $0.56(8)$ & $1.33(5)$ & $1.09(67)$ & $8.43 / 1.91$ \\
\hline $60022-01-01-02$ & 51998.78 & $1.01(3)$ & $2.57(1)$ & $-0.04(1)$ & $11.92(3)$ & $1.2(1)$ & $3.26(2)$ & $6.59(5)$ & $0.58(7)$ & $1.33(7)$ & 0.87 & $8.43 / 1.91$ \\
\hline $60022-01-01-03$ & 51999.78 & $1.02(7)$ & $2.47(3)$ & $0.04(1)$ & $12.31(4)$ & $1.20(3)$ & $2.39(4)$ & $6.55(4)$ & $0.55(7)$ & $1.57(6)$ & $1.15(67)$ & $8.02 / 1.69$ \\
\hline $60022-01-02-00$ & 52028.61 & $1.00(9)$ & $2.51(6)$ & $0.03(1)$ & & $1.20(5)$ & $2.74(4)$ & $6.56(2)$ & $0.53(6)$ & $1.22(5)$ & & $7.56 / 1.62$ \\
\hline $60022-01-02-01$ & 52028.94 & & $2.54(2)$ & $0.01(1)$ & & $1.20(6)$ & $2.51(6)$ & $6.50(6)$ & $0.59(5)$ & $1.23(3)$ & $1.05(67)$ & $7.62 / 1.65$ \\
\hline $60022-01-01.04$ & 52029.47 & $1.07(3)$ & $2.52(1)$ & $0.06(2)$ & $13.88(6)$ & $1.17(9)$ & $3.15(2)$ & $6.50(7)$ & $0.62(7)$ & $1.61(8)$ & $0.98(67)$ & $9.15 / 2.02$ \\
\hline $60022-01-01-05$ & 52029.61 & $1.03(6)$ & $2.51(2)$ & $0.2(1)$ & $13.36(3)$ & $1.20(5)$ & $2.39(6)$ & $6.51(6)$ & $0.50(8)$ & $1.03(5)$ & & $8.80 / 2.21$ \\
\hline $60022-01-01-06$ & 52029.69 & & & $-0.01(1)$ & & $1.20(4)$ & $2.84(9)$ & $6.54(2)$ & $0.53(9)$ & $1.26(6)$ & $1.40(67)$ & $7.57 / 1.58$ \\
\hline $60022-01-03-00$ & 52067.39 & $1.01(4)$ & $2.53(1)$ & $0.21(7)$ & $13.35(3)$ & $1.20(7)$ & $2.62(4)$ & $6.51(2)$ & $0.5(1)$ & $1.22(3)$ & $1.09(67)$ & $8.87 / 2.22$ \\
\hline $60022-01-03-01$ & 52067.58 & $1.03(5)$ & $2.52(3)$ & $0.04(1)$ & $12.65(3)$ & $1.20(4)$ & $2.36(5)$ & $6.55(8)$ & $0.35(4)$ & $1.28(3)$ & $1.30(67)$ & $8.22 / 1.82$ \\
\hline $60022-01-03-01$ & 52068.30 & $1.07(9)$ & $3.16(2)$ & $-0.46(9)$ & $10.82(4)$ & $1.20(5)$ & & & & $1.58(4)$ & $1.20(67)$ & $8.24 / 1.94$ \\
\hline $60022-01-04-03$ & 52102.363 & $1.03(9)$ & $3.25(9)$ & $-0.57(8)$ & $9.44(7)$ & $1.31(7)$ & $5.47(4)$ & $7.07(3)$ & $0.50(9)$ & $1.64(5)$ & $1.07(67)$ & $7.44 / 1.60$ \\
\hline $60022-01-04-00$ & 52103.361 & $1.01(5)$ & $2.72(5)$ & $-0.20(8)$ & $9.7(1)$ & $1.30(6)$ & $6.65(9)$ & $7.09(4)$ & $0.52(9)$ & $1.86(5)$ & $1.24(67)$ & $8.38 / 1.78$ \\
\hline $60022-01-04-02$ & 52103.685 & $1.0(1)$ & $2.87(6)$ & $-0.1(1)$ & $10.68(9)$ & $1.20(7)$ & $4.01(9)$ & & & & & $7.78 / 1.72$ \\
\hline $60022-01-01-07$ & 52127.54 & $0.99(3)$ & $2.53(7)$ & $0.24(1)$ & $13.08(3)$ & $1.18(4)$ & $2.64(9)$ & $6.58(3)$ & $0.36(8)$ & $1.14(3)$ & $1.10(67)$ & $8.71 / 2.21$ \\
\hline $60022-01-03-03$ & 52128.667 & $0.99(5)$ & $2.78(5)$ & $-0.26(5)$ & $10.23(1)$ & $1.20(8)$ & $6.8(1)$ & $6.34(5)$ & $0.4(1)$ & $1.57(3)$ & $1.04(67)$ & $8.24 / 1.94$ \\
\hline $60022-01-04-04$ & 52128.734 & $1.0(1)$ & $2.93(5)$ & $-0.29(8)$ & $10.40(7)$ & $1.20(9)$ & $5.8(1)$ & $7.09(4)$ & & $1.65(4)$ & & $8.04 / 1.96$ \\
\hline $60022-01-03-04$ & 52129.386 & $1.02(6)$ & $2.72(6)$ & $-0.17(7)$ & $9.9(1)$ & $1.20(4)$ & $5.97(9)$ & $6.42(3)$ & $0.37(8)$ & $1.83(3)$ & $1.01(67)$ & $8.38 / 1.86$ \\
\hline $60022-01-04-01$ & 52131.114 & $1.07(6)$ & $2.87(6)$ & $-0.3(1)$ & $9.36(3)$ & $1.20(8)$ & $4.56(6)$ & $7.09(5)$ & $0.46(6)$ & $1.64(4)$ & $1.15(67)$ & $7.15 / 1.53$ \\
\hline $60022-01-05-00$ & 52131.507 & $1.0(1)$ & $2.62(5)$ & $-0.09(1)$ & $8.5(1)$ & $1.30(6)$ & $5.8(1)$ & $5.99(7)$ & $0.47(4)$ & $1.69(3)$ & $1.16(67)$ & $7.49 / 1.60$ \\
\hline $60022-01-05-01$ & 52131.375 & $0.99(7)$ & $2.89(8)$ & $-0.36(1)$ & $8.2(1)$ & $1.20(4)$ & $6.5(1)$ & & $0.41(8)$ & $1.72(6)$ & $1.05(67)$ & $7.61 / 1.64$ \\
\hline $60022-01-06-01$ & 52166.493 & $1.0(1)$ & $2.75(3)$ & $-0.17(2)$ & $11.19(5)$ & $1.20(9)$ & $5.53(5)$ & $5.87(7)$ & $0.43(9)$ & $1.61(3)$ & $0.96(67)$ & $8.73 / 2.13$ \\
\hline $60022-01-06-03$ & 52168.754 & $1.00(7)$ & $2.96(6)$ & $-0.37(5)$ & $11.36(6)$ & $1.2(1)$ & $5.52(9)$ & $5.57(8)$ & $0.39(4)$ & $1.63(3)$ & $1.16(67)$ & $8.76 / 2.05$ \\
\hline $60022-01-06 m 05$ & 52169.610 & $1.01(4)$ & $2.65(4)$ & $0.08(7)$ & $11.04(7)$ & $1.20(5)$ & $5.26(8)$ & $6.17(3)$ & $0.418)$ & $1.65(6)$ & $1.06(67)$ & $8.34 / 2.31$ \\
\hline $60022-01-06-06$ & 52169.678 & $1.02(5)$ & $3.04(6)$ & $-0.40(3)$ & $12.77(8)$ & $1.20(9)$ & $5.22(7)$ & $6.28(2)$ & 0.3866 & $1.63(8)$ & $1.11(67)$ & $8.94 / 2.20$ \\
\hline $60022-01-06-07$ & 52170.534 & $1.03(5)$ & $2.6(1)$ & $-0.04(3)$ & $7.20(4)$ & $1.20(5)$ & $5.33(3)$ & $6.47(7)$ & $0.50(8)$ & $1.68(7)$ & $1.12(67)$ & $6.58 / 1.38$ \\
\hline $60022-01-06-00$ & 52170.983 & $1.00(5)$ & $2.65(7)$ & $-0.15(5)$ & $9.4(1)$ & $1.30(6)$ & $6.53(7)$ & $5.87(6)$ & $0.39(7)$ & $1.64(4)$ & $1.1(67)$ & $7.98 / 1.72$ \\
\hline $60022-01-06-09$ & 52171.194 & $1.06(7)$ & $2.86(5)$ & $-0.23(4)$ & $11.1(1)$ & $1.20(7)$ & $5.70(5)$ & $6.47(8)$ & $0.37(9)$ & $1.74(5)$ & $1.06(67)$ & $8.75 / 2.13$ \\
\hline $60022-01-06-08$ & 52172.176 & $1.04(4)$ & $2.99(8)$ & $-0.43(6)$ & $10.40(5)$ & $1.20(9)$ & $5.39(6)$ & $6.57(6)$ & $0.4(1)$ & $1.71(4)$ & $1.1(67)$ & $8.22 / 1.82$ \\
\hline $60022-01-06 \times 10$ & 52172.241 & $1.0(1)$ & $2.66(4)$ & $-0.25(5)$ & $10.03(4)$ & $1.20(5)$ & $6.26(8)$ & $6.43(7)$ & $0.40(8)$ & $1.68(3)$ & $0.95(67)$ & $8.44 / 2.02$ \\
\hline $60022-01-06-11$ & 52172.725 & $0.99(6)$ & $2.62(9)$ & $-0.49(7)$ & $9.34(5)$ & $1.30(6)$ & $6.34(5)$ & $6.40(3)$ & $0.38(7)$ & $1.74(2)$ & $1.08(67)$ & $8.30 / 1.83$ \\
\hline $60022-01-06-12$ & 52172.792 & $1.00(3)$ & $3.41(9)$ & $-0.54(4)$ & $2.08(7)$ & $1.20(8)$ & $5.24(9)$ & $6.81(5)$ & $0.35(8)$ & $1.81(5)$ & $1.01(67)$ & $8.84 / 2.15$ \\
\hline $60022-01-06-13$ & 52172.852 & $1.03(5)$ & $2.83(3)$ & $-0.20(3)$ & $11.63(6)$ & $1.20(5)$ & $6.09(9)$ & $6.17(7)$ & $0.32(5)$ & $1.77(7)$ & $1.05(67)$ & $8.88 / 2.24$ \\
\hline $60022-01-07-00$ & 52201.445 & $1.04(4)$ & $2.75(5)$ & $-0.13(3)$ & $10.13(3)$ & $1.20(6)$ & $5.50(8)$ & $6.24(8)$ & $0.35(8)$ & $1.69(3)$ & $1.07(67)$ & $8.06 / 1.98$ \\
\hline
\end{tabular}


Table 4

(Continued)

\begin{tabular}{|c|c|c|c|c|c|c|c|c|c|c|c|c|}
\hline $\begin{array}{l}\text { Observational } \\
\text { ID }\end{array}$ & $\begin{array}{l}\text { MJD, } \\
\text { (day) }\end{array}$ & $\begin{array}{c}\alpha= \\
\Gamma-1\end{array}$ & $\begin{array}{c}T_{e^{*}} \\
(\mathrm{keV})\end{array}$ & $\log (A)$ & $N_{\mathrm{COM}^{b}}$ & $\begin{array}{c}T_{s}, \\
(\mathrm{keV})\end{array}$ & $N_{\text {Bbødy }}{ }^{b}$ & $\begin{array}{l}\text { Eline } \\
(\mathrm{keV})\end{array}$ & $\begin{array}{l}o_{\text {line }}, \\
(\mathrm{keV})\end{array}$ & $N_{\text {line }}{ }^{b}$ & $x_{\text {red }}^{2}($ dof $)$ & $F_{1} / F_{2}{ }^{\mathrm{c}}$ \\
\hline $60022-01-07-02$ & 52204.424 & $1.06(9)$ & $2.75(5)$ & $-0.23(6)$ & $9.11(4)$ & $1.20(5)$ & $5.42(9)$ & $6.36(6)$ & $0.3(1)$ & $1.65(4)$ & $0.89(67)$ & $7.57 / 1.67$ \\
\hline $60022-01-07-03$ & 52207.202 & $1.03(5)$ & $2.76(3)$ & $-0.02(1)$ & $11.05(5)$ & $1.20(7)$ & $5.07(9)$ & $5.85(7)$ & $0.35(8)$ & $1.62(3)$ & $1.15(67)$ & $8.31 / 2.32$ \\
\hline $60022-01-07-04$ & 52207.071 & $1.01(4)$ & $2.69(3)$ & $-0.01(1)$ & $10.37(3)$ & $1.20(3)$ & $5.56(8)$ & $6.52(5)$ & $0.4(1)$ & $1.76(5)$ & $1.12(67)$ & $8.26 / 2.06$ \\
\hline $60022-01-08-00$ & 52230.164 & $1.0(5)$ & $2.58(4)$ & $-0.01(1)$ & $7.64(1)$ & $1.3(1)$ & $5.67(7)$ & $5.87(7)$ & $0.50(9)$ & $1.64(3)$ & $1.15(67)$ & $6.84 / 1.49$ \\
\hline $60022-01-09-00$ & 52295.306 & $1.0(4)$ & $2.51(4)$ & $0.23(1)$ & $12.25(4)$ & $1.2(1)$ & $3.53(8)$ & $5.97(8)$ & $0.46(8)$ & $1.67(6)$ & $1.14(67)$ & $8.55 / 2.04$ \\
\hline $60022-01-10-00$ & 52321.559 & $1.0(5)$ & $2.50(4)$ & $0.11(1)$ & $13.26(4)$ & $1.20(9)$ & $2.71(8)$ & $5.85(9)$ & $0.41(6)$ & $1.71(8)$ & $1.2(67)$ & $8.72 / 1.97$ \\
\hline $60022-01-10-01$ & 52320.301 & $1.0(6)$ & $2.51(2)$ & $0.09(1)$ & $13.77(1)$ & $1.20(6)$ & $2.61(8)$ & $5.78(7)$ & $0.46(7)$ & $1.61(4)$ & $1.1(67)$ & $9.08 / 2.18$ \\
\hline $60022-01-10-02$ & 52320.876 & $1.0(3)$ & $2.52(1)$ & $0.06(1)$ & $12.19(4)$ & $1.20(5)$ & $2.60(8)$ & $5.87(6)$ & $0.4(1)$ & $1.65(4)$ & $1.04(67)$ & $8.01 / 1.76$ \\
\hline $60022-01-10-03$ & 52324.647 & $0.99(2)$ & $2.44(2)$ & $0.14(5)$ & $11.36(4)$ & $1.10(8)$ & $1.57(6)$ & $6.26(6)$ & $0.47(9)$ & $1.87(3)$ & $1.11(67)$ & $6.39 / 1.37$ \\
\hline $60022-01-10-04$ & 52325.760 & $0.99(2)$ & $2.44(3)$ & $0.15(6)$ & $11.91(5)$ & $1.10(9)$ & $2.23(6)$ & $6.47(7)$ & $0.51(6)$ & $1.79(6)$ & $1.09(67)$ & $6.39 / 1.37$ \\
\hline $60022-01-08-00$ & 52230.164 & $1.00(5)$ & $2.58(4)$ & $-0.02(1)$ & $7.64(2)$ & $1.2(1)$ & $5.67(7)$ & $6.75(6)$ & $0.53(8)$ & $1.86(4)$ & $0.88(67)$ & $6.84 / 1.49$ \\
\hline $60022-01-11-00$ & 52356.747 & $0.99(3)$ & $2.50(2)$ & $0.08(7)$ & $9.94(6)$ & $1.10(8)$ & $1.29(6)$ & $6.55(4)$ & $0.49(6)$ & $1.86(7)$ & $1.2(67)$ & $6.39 / 1.37$ \\
\hline $60022-01-11-01$ & 52356.814 & $0.99(1)$ & $2.47(1)$ & $0.12(4)$ & $11.79(3)$ & $1.10(5)$ & $1.58(6)$ & $6.46(3)$ & $0.5(1)$ & $1.85(7)$ & $1.15(67)$ & $7.54 / 1.64$ \\
\hline $60022-01-11-03$ & 52357.083 & $0.99(2)$ & $2.41(1)$ & $0.6(1)$ & $13.29(4)$ & $1.10(8)$ & $2.8(1)$ & $6.57(7)$ & $0.50(9)$ & $1.87(4)$ & $1.1(67)$ & $8.56 / 1.94$ \\
\hline $60022-01-11-02$ & 52394.572 & $1.0(1)$ & $2.51(2)$ & $0.41(4)$ & $12.56(4)$ & $1.10(4)$ & $6.2(1)$ & $6.38(5)$ & $0.39(7)$ & $1.94(3)$ & $1.19(67)$ & $9.92 / 2.36$ \\
\hline $60022-01-12-00$ & 52394.891 & $1.0(1)$ & $2.73(4)$ & $0.08(7)$ & $13.26(7)$ & $1.10(7)$ & $5.76(8)$ & $6.49(9)$ & $0.37(8)$ & $1.85(6)$ & $1.01(67)$ & $9.92 / 2.36$ \\
\hline $60022-01-13-00$ & 52554.152 & $1.0(1)$ & $2.46(2)$ & $0.6(1)$ & $12.5(9)$ & $1.1(1)$ & $9.48(4)$ & $6.35(5)$ & $0.35(4)$ & $1.91(4)$ & $1.07(67)$ & $9.92 / 2.36$ \\
\hline $60022-01-13-01$ & 52554.484 & $1.00(5)$ & $2.56(5)$ & $0.03(1)$ & $13.6(7)$ & $1.20(5)$ & $2.85(7)$ & $6.48(7)$ & $0.36(9)$ & $1.86(3)$ & $1.16(67)$ & $8.87 / 1.99$ \\
\hline $90022-05-01-00$ & 53108.101 & $1.04(8)$ & $2.42(9)$ & $-0.18(6)$ & $4.22(3)$ & & & & & $0.87(5)$ & $1.36(67)$ & $3.85 / 0.79$ \\
\hline $90022-05-01-01$ & 53108.256 & $1.00(8)$ & $3.1(1)$ & $-0.46(7)$ & $4.75(7)$ & $1.59(9)$ & $3.68(5)$ & $6.34(5)$ & $0.60(8)$ & $1.03(7)$ & $1.38(67)$ & $3.99 / 0.84$ \\
\hline $90022-05-02-00$ & 53238.243 & $1.01(8)$ & $2.56(4)$ & $0.10(6)$ & $4.20(7)$ & $1.19(7)$ & $2.58(9)$ & $6.39(9)$ & $0.62(3)$ & $0.77(9)$ & $1.34(67)$ & $3.07 / 0.64$ \\
\hline $90022-05-03-00$ & 53245.990 & $1.06(9)$ & $2.36(2)$ & $0.30(6)$ & $5.42(5)$ & $1.19(8)$ & & & & $0.79(8)$ & & $3.76 / 0.75$ \\
\hline $90022-05-04-00$ & 53252.490 & $1.00(4)$ & $2.5(1)$ & $0.10(7)$ & $6.05(9)$ & $1.19(6)$ & $1.51(8)$ & $6.6(1)$ & $0.65(8)$ & $0.86(9)$ & $1.00(67)$ & $3.11 / 0.83$ \\
\hline $90022-05-04-01$ & 53253.859 & $1.0(1)$ & $2.47(2)$ & $0.06(2)$ & $6.08(3)$ & $1.50(6)$ & $2.49(5)$ & $6.50(9)$ & $0.66(6)$ & $0.83(5)$ & $1.25(67)$ & $4.23 / 0.85$ \\
\hline $90022-05-05-00$ & 53263.468 & $1.01(4)$ & $2.52(1)$ & $0.04(1)$ & $5.33(2)$ & $1.50(8)$ & $2.24(2)$ & $6.49(7)$ & $0.67(8)$ & $0.97(7)$ & $1.15(67)$ & $4.23 / 0.85$ \\
\hline $90022-05-06-00$ & 53265.412 & $1.09(6)$ & $2.52(2)$ & $0.01(1)$ & $5.42(3)$ & $1.45(7)$ & $2.46(3)$ & $6.6(1)$ & $0.63(6)$ & $0.87(5)$ & $1.15(67)$ & $3.78 / 0.75$ \\
\hline $90022-05-06-01$ & 53265.541 & $1.0(1)$ & $2.50(1)$ & $0.01(1)$ & $5.56(5)$ & $1.39(5)$ & $2.08(4)$ & $6.62(9)$ & $0.62(4)$ & $0.79(7)$ & $1.05(67)$ & $3.79 / 0.77$ \\
\hline $90022-05-06-02$ & 53265.675 & $1.01(2)$ & $2.51(2)$ & $0.04(2)$ & $5.54(6)$ & $1.41(4)$ & $2.34(6)$ & $6.67(5)$ & $0.61(8)$ & $0.79(5)$ & $1.07(67)$ & $3.82 / 0.78$ \\
\hline $90022-05-06-03$ & 53265.806 & $1.02(5)$ & $2.56(2)$ & $-0.02(1)$ & $4.98(3)$ & $1.6(1)$ & $2.22(5)$ & $6.6(1)$ & $0.62(9)$ & $0.81(9)$ & $1.08(67)$ & $3.44 / 0.69$ \\
\hline $90022-05-07-00$ & 53279.124 & $1.00(7)$ & $2.53(1)$ & $0.10(6)$ & $3.53(1)$ & $1.70(8)$ & $2.45(3)$ & $6.64(7)$ & $0.64(5)$ & $0.85(6)$ & $1.18(67)$ & $3.44 / 0.69$ \\
\hline $90022-05-07-00$ & 53280.960 & $1.03(9)$ & $2.42(1)$ & $0.31(3)$ & $5.42(3)$ & $1.50(9)$ & $2.18(3)$ & $6.65(8)$ & $0.62(8)$ & $0.79(7)$ & $0.97(67)$ & $3.44 / 0.69$ \\
\hline $94307 \sim 05 \sim 01-00$ & 55129.659 & $1.02(3)$ & $3.16(4)$ & $0.41(3)$ & $4.53(7)$ & $1.4(1)$ & $3.08(2)$ & $6.58(2)$ & $0.4(1)$ & $0.32(1)$ & $1.09(67)$ & $3.21 / 1.28$ \\
\hline $94307-05-01-000$ & 55440.307 & $1.04(4)$ & $3.16(5)$ & $0.45(4)$ & $4.53(7)$ & $1.50(8)$ & $3.08(3)$ & $6.59(5)$ & $0.40(7)$ & $0.34(2)$ & $0.87(67)$ & $3.21 / 1.28$ \\
\hline $94307-05-01-00$ & 55440.62 & $1.03(8)$ & $2.50(2)$ & $0.25(2)$ & $4.67(2)$ & $1.4(1)$ & $4.17(4)$ & $6.64(9)$ & $0.62(9)$ & $0.68(9)$ & $1.13(67)$ & $3.78 / 0.78$ \\
\hline $94307-05-01-01$ & 55440.701 & $1.03(9)$ & $3.16(4)$ & $0.41(3)$ & $4.53(7)$ & $1.40(6)$ & $3.08(2)$ & $6.58(3)$ & $0.4(1)$ & $0.32(6)$ & $0.89(67)$ & $3.21 / 1.28$ \\
\hline $94307-05-01-02$ & 55440.766 & $1.02(3)$ & $3.26(7)$ & $-0.56(3)$ & $4.57(1)$ & $1.35(7)$ & $4.13(2)$ & $6.21(2)$ & $0.5(1)$ & $0.21(7)$ & $0.98(67)$ & $3.21 / 1.28$ \\
\hline
\end{tabular}

Notes. Parameter errors correspond to $1 \sigma$ confidence level.

The spectral model is wabs $*$ (blackbody + COMPTB + Gaussian), where $N_{\mathrm{H}}$ is fixed at a value $1.6 \times 10^{22} \mathrm{~cm}^{-2}($ Oosterbroek et al. 2001$)$; color temperature $T_{\mathrm{BB}}$ is fixed at $0.6 \mathrm{keV}$ (see comments in the text).

${ }^{b}$ Normalization parameters of blackbody and COMPTB components are in units of $L_{37}^{\text {soft }} / d_{10}^{2}$, where $L_{37}^{\text {soft }}$ is the source luminosity in units of $10^{37}$ erg $s^{-1}, d_{10}$ is the distance to the source in units of $10 \mathrm{kpc}$, and Gaussian component is in units of $10^{-2} \times$ total photons cm $\mathrm{cm}^{-2} \mathrm{~s}^{-1}$ in line.

${ }^{c}$ Spectral fuxes $\left(F_{1} / F_{2}\right)$ in units of $\times 10^{-9} \mathrm{erg} \mathrm{s}^{-1} \mathrm{~cm}^{-2}$ for $(3-10)$ and $(10-50) \mathrm{keV}$ energy ranges, respectively.

telescopes allows us to determine the parameters of blackbody components at low energies. Thus, in order to fit the RXTE data we have to fix the temperature of blackbody component at a value of $k T_{\mathrm{BB}}=0.6 \mathrm{keV}$ obtained as an upper limit in our analysis of the BeppoSAX data. The best-fit spectral parameters using RXTE observations are presented in Table 4 . In particular, we find that electron temperature $k T_{e}$ of the COMPTB component varies from 2.3 to $4.5 \mathrm{keV}$, while the photon index $\Gamma$ is almost constant ( $\Gamma=1.99 \pm 0.02)$ for all observations. It is worth noting that the width $\sigma_{\text {line }}$ of Gaussian component does not vary significantly and it is in the range of $0.5-0.8 \mathrm{keV}$.

Color temperature $k T_{s}$ of COMPTB component changes from $1.2 \mathrm{keV}$ to $1.7 \mathrm{keV}$, which is consistent with that using the BeppoSAX data set of our analysis (see Table 3 ) and previous studies by Oosterbroek et al. (2001), den Hartog et al. (2003), and Chenevez et al. (2006). We should also emphasize that the temperature of the seed photons $k T_{s}$ of the COMPTB component usually increases up to $1.7 \mathrm{keV}$ in the fainter phases and generally decreases to $1.2 \mathrm{keV}$ in the bright phases.
In Figure 4, we show an example of the best-fit RXTE spectrum of GX $3+1$ for the fainter luminosity phases and the residuals (bottom panel) with $\Delta \chi$ for the 94307-05-01-000 observation. Blue, red, and purple lines stand for blackbody, COMPTB, and Gaussian components, respectively.

In Figure 5, we also show examples of typical photon spectra $E * F(E)$ spectral diagrams of GX $3+1$ during the fainter phase (94307-05-01-00, blue) and the brighter phases (6002201-13-01, red) detected with RXTE on MJD 55440.62 and 52544.48 , respectively. The adopted spectral model shows a very good fidelity throughout all data sets used in our analysis. Namely, a value of reduced $\chi_{\text {red }}^{2}=\chi^{2} / N_{\text {dof }}$, where $N_{\text {dot }}$ is a number of degree of freedom, is less or about 1.0 for most observations. For a small fraction (less than $2 \%$ ) of spectra with high counting statistics $\chi_{\text {red }}^{2}$ reaches 1.5 . However, it never exceeds our rejection limit of 1.7 . Note that the energy range for the cases, in which we obtain the poor fit statistic (two among 101 spectra with $\chi^{2}=1.7$ for 67 dof), is related to the iron line region. It is possible that the shape of iron line is more complex 
than a simple Gaussian (i.e., a blend of different energies, presence of the edge, or broadening by Comptonization). The fits tend to favor a broad line (see Table 4), which might be caused by Comptonization. However, this possible complexity is not well constrained by our data.

It is worth noting that we find some differences between our values of the best-fit model parameters and those in the literature. In particular, the photon index $\Gamma$, estimated by Oosterbroek et al. ( 2001 ) for observation id $=20835001$, is $1.60 \pm 0.25$. This discrepancy in index values can be a result of using slightly different spectral models by us and Oosterbroek et al. (2001).

Thus using the broadband BeppoSAX observations we can accurately determine all of the parameters of our spectral model while using the extensive observations of GX $3+1$ by RXTE we are able to investigate the overall pattern of the source behavior during the spectral transitions in the $3-50 \mathrm{keV}$ energy range.

\section{OVERALL PATTERN OF X-RAY PROPERTIES}

\section{I. Evolution of X-Ray Spectral Properties During Transitions}

As was mentioned above, at timescales larger that one minute, GX $3+1$ exhibits two kinds of variability, slow and mild. The former one (slow) has a timescale of order years. This slow variability are seen in the faint phases and bright phases which are related to low and high luminosities, respectively. On the other hand the mild variability has a timescale of order of days and modulation depth in the $3-10 \mathrm{keV}$ band is typically $20 \%$. The ASM $(2-12 \mathrm{keV})$ mean flux correlates with COMPTB normalization $\left(N_{\mathrm{COM}}\right)$ and tends to anticorrelate with the electron plasma temperature of $\mathrm{CC} T_{e}$ (see Figure 6). Such mild variability is detected for both the fainter and brighter phases for GX $3+1$. It should be noted that the COMPTB normalization changes are larger in the bright phase than that during the faint phase, while the electron temperature $T_{e}$ variations are almost the same for both phases.

One can relate slow and mild variabilities of $\mathrm{GX} 3+1$ to slow and mild changes of mass accretion rate, respectively. The slow variability by factor four has been seen in the 1996-2010 observations by ASM/RXTE. The same kind of changes of the flux was also observed in the earlier observations by Makishima et al. (1983). In turn, in the next section it will be shown that the slow variability can be related to transitions between the brighter and fainter phases along with small variations of the electron temperature $k T_{e}$.

We found that the X-ray spectra of GX $3+1$ over the bright and faint phases are quite stable. Moreover, we detected a constant $3-50 \mathrm{keV}$ spectral shape over the slow and mild variability stages. The best-fit parameters are listed in Table 4 . The faint/ bright phase transitions are characterized by the spectra with an almost constant spectral index $\alpha$ of 1 or photon index $\Gamma$ of 2 (see Figure 7 ). We have also established common characteristics of the rise-decay spectral transition of GX $3+1$ based on their spectral parameter evolution of X-ray emission in the energy range from 3 to $50 \mathrm{keV}$ using $\mathrm{PCA} /$ RXTE data. In Figures 4 and 5 we present typical examples of the RXTE bright and faint phase spectra for $\mathrm{GX} 3+1$. In fact, one can clearly see from these figures that the normalization of the thermal component is a factor of two higher in the bright phase than that in the faint phase, although the photon indices $\Gamma$ for each of these spectra are concentrated around 2 (see Figures 6 and 7 ).

In fact, we test the hypothesis of $\Gamma_{\text {appr }} \approx 2$ using $\chi^{2}$. statistic criterion. We calculate the distribution of $\chi_{\text {red }}^{2}\left(\Gamma_{\text {appr }}\right)=$ $\frac{1}{N} \sum_{i=1}^{N}\left(\Gamma_{i}-\Gamma_{\text {appr }} / \Delta \Gamma_{i}\right)^{2}$ versus of $\Gamma_{\text {appr }}$ and we find a sharp

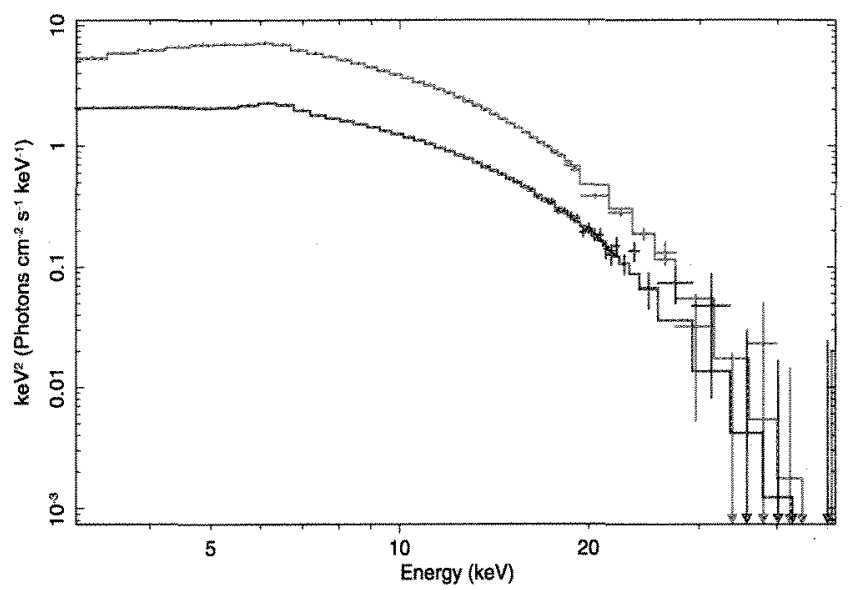

Figure 5. Examples of X-ray spectra, presented as $E * F(E)$ spectral diagrams, of GX $3+1$ during faint phase (94307-05-01-00, blue) and bright phase (60022-0113-01, red) detected with RXTE on MJD 55440.62 and 52544.48 , respectively.

(A color version of this figure is available in the online joumal.)

minimum of function $\chi_{\text {red }}^{2}\left(\Gamma_{\text {appr }}\right)$ around 1 which takes place in the range of $\Gamma_{\text {appr }}=1.99 \pm 0.01$ with a confidence level of $67 \%$ and $\Gamma_{\text {appr }}=1.99 \pm 0.02$ with a confidence level $99 \%$ for $101 \mathrm{dof}$ (see the similar figure of $\chi_{\text {red }}^{2}\left(\Gamma_{\text {appr }}\right.$ ) for $4 U$ 1728-34 in ST11). Using BeppoSAX data FT11 suggested that the photon index $\Gamma$ is approximately 2 for many NS binaries which are observed in different spectral states. FT1 1 characterize the spectral state by a value of electron temperature $T_{e}$ and they show that $\Gamma=2 \pm 0.2$ (or $\alpha=1 \pm 0.2$ ) when $k T_{e}$ changes from 2.5 to $25 \mathrm{keV}$.

A number of X-ray flaring episodes of $\mathrm{GX} 3+1$ has been detected with RXTE during 2001-2002 ( $R 4$ set) with a good rise-decay coverage. We have searched for common spectral and timing features which can be revealed during these spectral transition episodes. We present the combined results of the spectral analysis of these observations using our spectral model wabs $*$ (blackbody + COMPTB + Gaussian $)$ in Figures 6 and 7 . ASM/RXTE count rate is shown on the top panel of these figures. Further, from the top to the bottom, we show the model flux in two energy bands $3-10 \mathrm{keV}$ (blue points) and $10-50 \mathrm{keV}$ (crimson points). In the next panel we show a change of the TL electron temperature $k T_{e}$. One can clearly see the low amplitude spectral transition on timescales of $\sim 1-2$ days from the brighter phase to the fainter phase during the time period from MJD 52000 to MJD 52200 while $k T_{e}$ only varies from $2.3 \mathrm{keV}$ to $4.5 \mathrm{keV}$ during this transition.

Normalizations of the COMPTB and blackbody component (crimson and blue points, respectively) are shown in the next panel of Figures 6 and 7 . In particular, one can see from Figures 6 and 7 how the COMPTB normalization $N_{C O M}$ correlates with the variations of ASM count rate and the model flux in $3-10 \mathrm{keV}$ energy band. On the other hand, the normalization of the blackbody component $N_{\mathrm{BB}}$ is almost constant except at the mild variability episode peak, when $N_{\mathrm{BB}}$ increases from 0.02 to 0.14 (see blue points in Figure 6 at MJD $=52130$ and 52170 ). Moreover, these spectral variability transitions are related to a noticeable increase of flux in the $3-10 \mathrm{keV}$ energy range and corresponding decrease of flux that takes place in the $10-50 \mathrm{keV}$ energy range (see the second panels from above in Figures 6 and 7 ).

The illumination fraction $f$ varies from 0.1 to 0.9 as seen from Figure 8 while the index $\alpha$ only slightly varies with time around 1 (or $\Gamma \sim 2$ ) (see Figures 6 and 7 ). However in most cases 


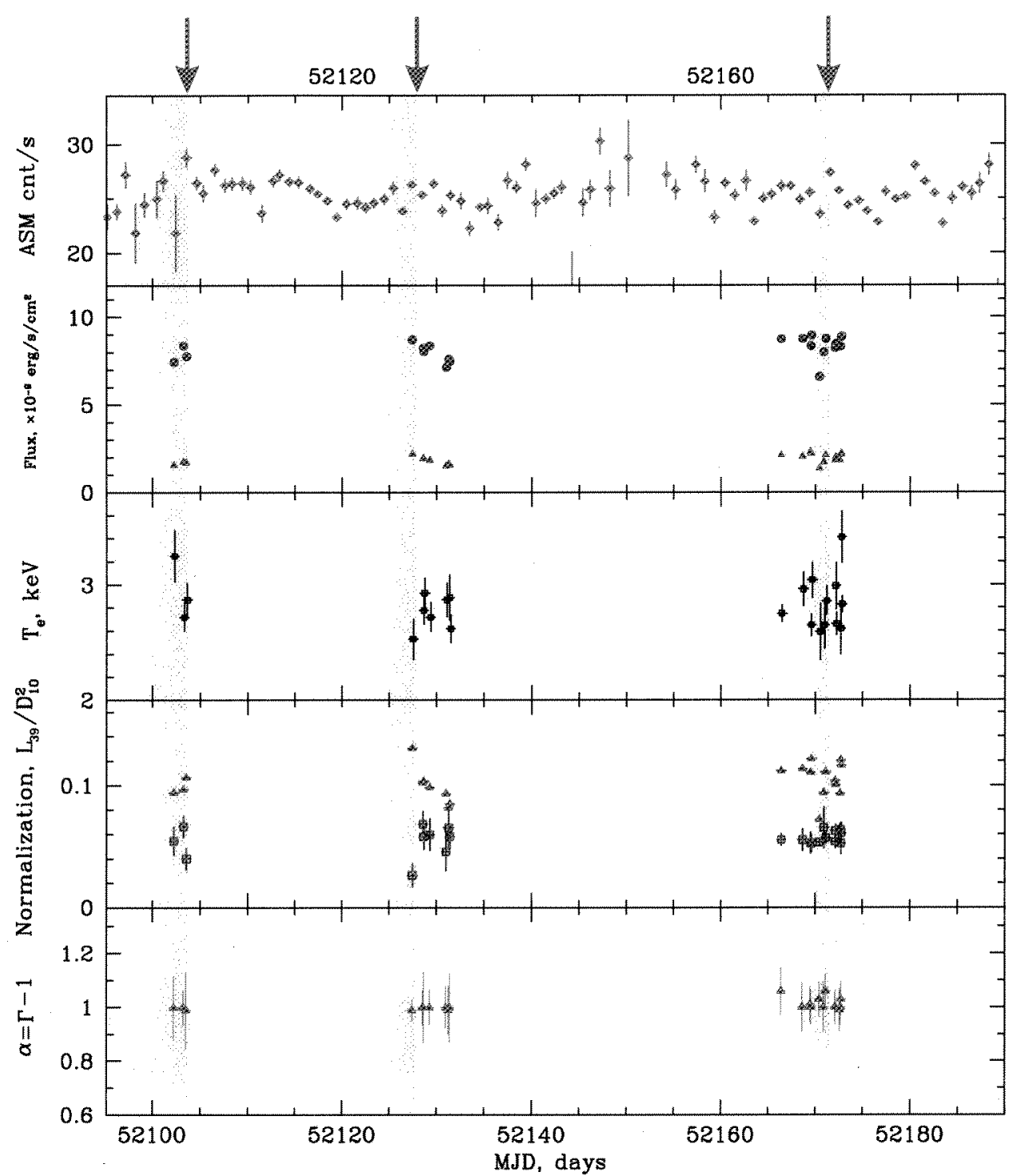

Figure 6. From top to bottom: evolutions of the ASM/RXTE count rate, model flux in 3-10 keV and 10-60 keV energy ranges (blue and crimson points, respectively), electron temperature $T_{e}$ in $\mathrm{keV}, C O M P T B$ and blackbody normalizations (crimson and blue points, respectively), and spectral index $\alpha=\Gamma-1$ during 2001-2002 outburst transition set $(R 4)$. The rising phases of the mild transition are marked with blue vertical strips. The peak burst times are indicated by the arrows on the top of the plot.

(A color version of this figure is available in the online journal.)

the soft disk radiation of $\mathrm{GX} 3+1$ is subjected to reprocessing in a $\mathrm{CC}$ and only some fraction of the soft photon emission component $(1-f)$ is directly seen by the Earth observer. Note that $f$ changes with COMPTB normalization (see Figure 8 , the inner panel in the left-hand upper corner). The energy spectrum of GX $3+1$ during almost all states is dominated by a Comptonized component while the direct soft photon emission is always weaker and detectable in the flaring episodes only (see also Figures 6 and 7 ).

Note that for BHs a definition of spectral transition involves a change of photon index $\Gamma$ (see, e.g., ST09). However there is no one-to-one correspondence between $\Gamma$ and cutoff (or $e$-fold) energy $E_{\text {fold }}$. Titarchuk \& Shaposhnikov (2010) demonstrate using RXTE data for BH binary XTE J1550-564 that $E_{\text {fold }}$ decreases when $\Gamma$ increases from 1.4 to $2.1-2.2$ until $\Gamma$ reaches 2.2 and then $E_{\text {fold }}$ increases. Thus for a $B H$ the main parameter used for the spectral transition definition is a variable photon index $\Gamma$ which monotonically increases when the source goes into the bright phase.

It is important to emphasize once again that in the NS binary GX $3+1$ the transition from the faint phase to the bright phase takes place when COMPTB normalization $N_{\text {COM }}=L_{39}^{\text {soft }} / D_{10}^{2}$ changes from 0.04 to 0.15 . Thus, we define the NS spectral transition in terms of the COMPTB normalization. In this case the faint phase is characterized by the low normalization value while the bright phase is related to the high normalization value. In Figure 9, we demonstrate the dependence of COMPTB normalization $L_{39}^{\text {sott }} / D_{10}^{2}$ on $k T_{e}$ using these best-fit parameters for GX $3+1$ and 4 U 1728-34 for the BeppoSAX and RXTE data. From this figure one can clear see a monotonic behavior $N_{\mathrm{COM}}$ versus $k T_{e}$, namely, $k T_{e}$ decreases when the soft flux increases. It is worth noting that the $k T_{e}$ values obtained using $\mathrm{GX} 3+1$ data 


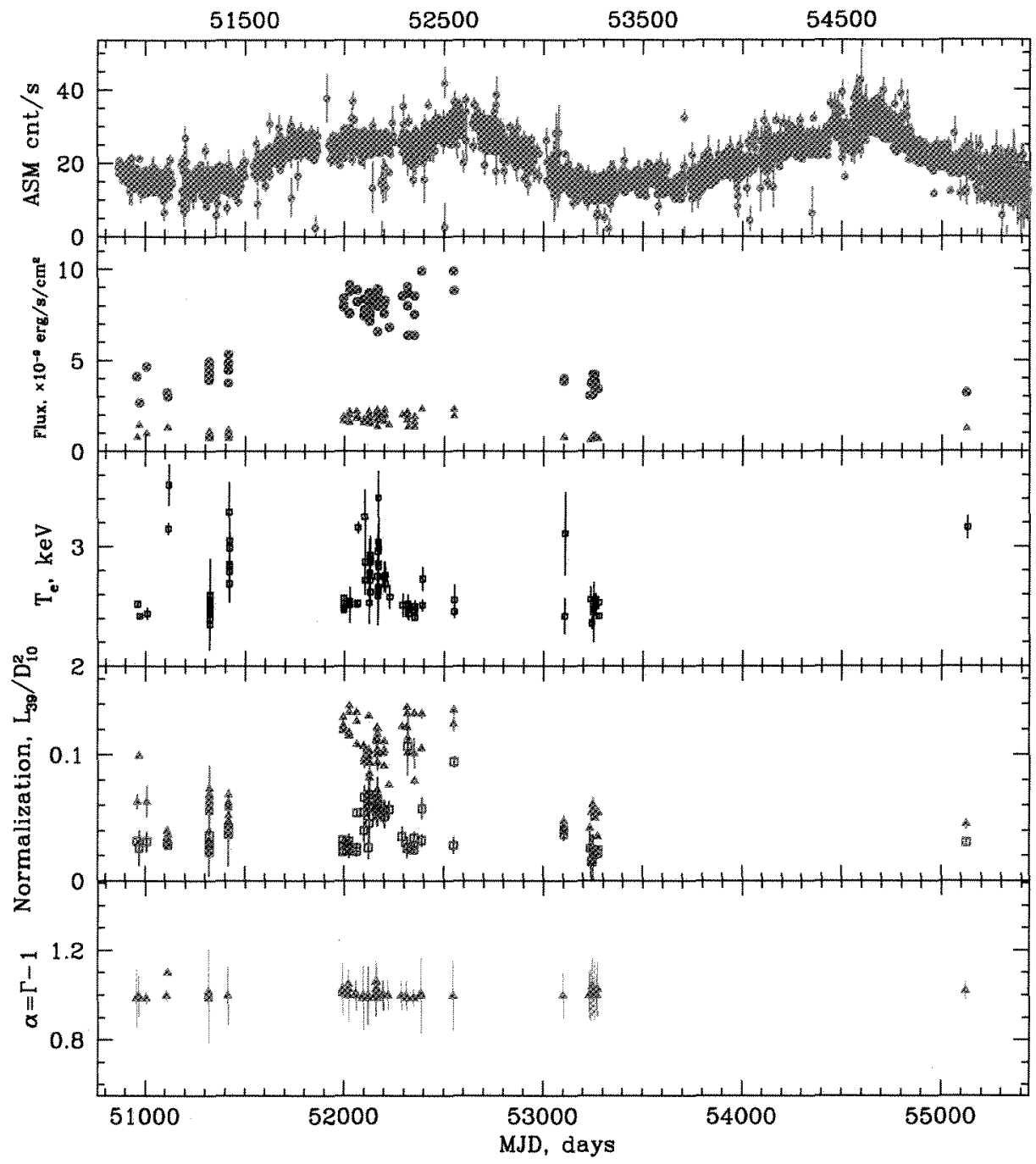

Figure 7. Same to that presented in Figure 6 but for all the RXTE sets $(R I-R 7)$ to demonstrate slow $($ long - tem $)$ variability.

(A color version of this figure is available in the online journal.)

for BeppoSAX and $R X T E$ reach the asymptotic value of about $2.5 \mathrm{keV}$ at high values of the soft flux $\left(N_{\mathrm{COM}}>0.05\right)$.

To demonstrate transition properties of $\mathrm{GX} 3+1$ in terms of flux (or luminosity) we define the hard color (HC) as a ratio of the flux in the $10-50 \mathrm{keV}$ to that in the $3-50 \mathrm{keV}$ energy band, and the soft color (SC) as a ratio of the flux in the $3-10 \mathrm{keV}$ to that in the $10-50 \mathrm{keV}$ energy range. Plotting $\mathrm{HC}$ versus SC we created our color-color diagram (CD, see the left panel of Figure 10 ) for two atoll sources GX $3+1$ (pink) and 4U 1728-34 (blue). As it appears from this figure the tracks of these two sources display a smooth and continuous (monotonic) function, pointing out the similar physical mechanism of hard/soft flux transition during a long-term source evolution for these two objects. In Figure 10 (right panel), we demonstrate a fragment of ASM light curve of $\mathrm{GX} 3+1$ which illustrates two types of flux variability. The long-term time trend (from bright to faint) is related to COMPTB normalization changes and while the second one shows short-term time variations (UB-LB) related to the $\mathrm{CC}$ electron temperature variations. The blue line displays a mean count rate and indicates to long-term variability of GX $3+1$ flux. Note that the track of Figure 10 (left panel) reflects a long-term evolution of GX $3+1$.
It is worth noting that among all NSs only a few of atoll and $\mathrm{Z}$-sources demonstrate a full track on $\mathrm{CD}$ in a wide range of luminosity. For example, atoll sources, such as $4 U$ 1728-34, usually show a wide range of spectral states during transitions which are related to changes of the total luminosity and mass accretion rate. One can establish a substantial difference between a NS and a $\mathrm{BH}$ due to these flare episodes when a source evolves from the faint phase to the bright phase and when the plasma temperature of a Comptonized region changes remarkably (like in $4 \mathrm{U} 1728-34$ from $2.5 \mathrm{keV}$ to $15 \mathrm{keV}$ during IS $-B$ states). On the other hand GX $3+1$ shows significant changes in the total luminosity but with only a slight variation of electron temperature $k T_{e}$ in its banana state. However, the photon index $\Gamma$ stays around a value of two and is independent of the soft photon luminosity both in the faint phase and the bright phase.

\subsection{Timing Properties During $L B-U B$ Transitions}

The RXTE light curves have been analyzed using the powspec task from FTOOLS 5.1. The timing analysis PCA/RXTE data was performed in 13-30 keV energy range using the event mode. The time resolution for this mode is $1.2 \times 10^{-4} \mathrm{~s}$. We generated 

GX $3+1 / \mathrm{RXTE}$
GX $3+1 /$ BepposAX
4U 1728-34/RXTE
筑 4 U 1728-34/BeppoSAX

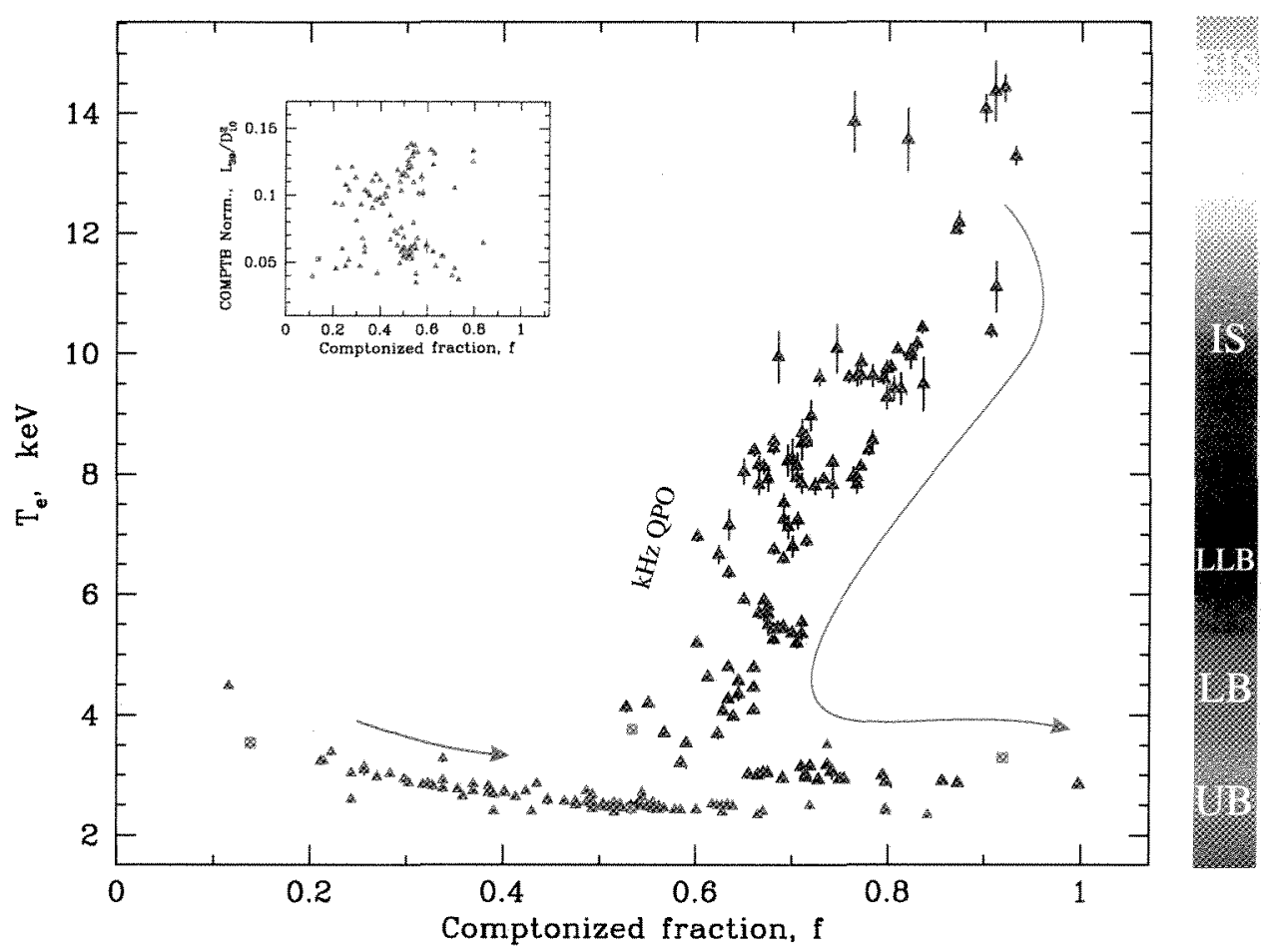

Figure 8. Electron temperature $T g_{e}$ (in keV) plotted vs. illumination fraction $f=A /(1+A)$ for atoll sources $\mathrm{GX} 3+1$ and $4 \mathrm{U} 1728-34$ during mild variability. Pink/bright blue and blue/green points correspond to RXTE/BeppoSAX observations of GX $3+1$ and 4 U 1728-34, respectively. For GX $3+1$ COMPTB normalization measured in $L_{39}^{\text {soft }} / D_{10}^{2}$ units vs. illumination fraction $f$ is plotted in the incorporated panel (top left) during long-term (slow) variability (see Table 4 ). The bended arrows are related to an increase of mass accretion rate. On the right-hand side we show a sequence of CD states (EIS: the extreme island state, IS: island state, LLB: lower left banana state, LB: lower banana state, and UB: upper banana state) which are listed according to the standard atoll-Z scheme (Hasinger \& van der Klis 1989). One can see that $T_{e}$ is directly related with the sequence of $\mathrm{CD}$ states. Along the track for $4 \mathrm{U} 1728-34$ we indicate points of $T_{e}-f$ correlation where $\mathrm{kHz}$ QPOs are detected.

(A color version of this figure is available in the online journal.)

power density spectra (PDS) in $0.1-500 \mathrm{~Hz}$ frequency range using light curves with $10^{-3} \mathrm{~s}$ time resolution. We subtracted the contribution due to Poissonian noise and Very Large Event Window for all PDSs. We used QDP/PLT plotting package to model PDS.

Previously, timing analysis of PCA/RXTE data for GX $3+1$ observed on 1999 August (our R3 set), when the sources was in $L B$ phase, was made by Oosterbroek et al. (2001). We investigated a timing behavior of $\mathrm{GX} 3+1$ for our data set during all transitions between $L B$ and $U B$ phases (see Figure 11 ). In particular, the power spectrum of $G \times 3+1$ consists of very low frequency noise (VLFN, described by a power law) and highfrequency noise (HFN, described by a cutoff power law; see van der Klis 2005).

In the LB phases (A red, 60022-01-13-01. MJD $=52554 ; A$ blue, 60022-01-01-00, MJD $=51998$ ) the best fit to the average power spectrum results in rms VLFN component of $2 \%$ (in the $0.1-1 \mathrm{~Hz}$ range) described by power-law $v^{-\alpha_{L F}}$ with the index of $\alpha_{\mathrm{LF}} \sim 1.7$, whereas HFN rms (in the $1-100 \mathrm{~Hz}$ range) has $4.7 \%$ with $\alpha_{\mathrm{HF}} \sim 1.0$ and $v_{\text {catoff }} \sim 30 \mathrm{~Hz}$. Generally the index of VLFN $\alpha_{\mathrm{LF}}$ gradually decreases from 1.7 to 1.4 toward UB. However in the vicinity of a transition point between $L B$ and UB (red histogram of panel (B) of Figure 11) $\alpha_{\text {LF jumps to } 1.8}$ $(\mathrm{B}$ red, 94307-05-01-00, $\mathrm{MJD}=55129)$ and decreases again to 1.4 ( $\mathrm{B}$ blue, 60022-01-11-03, $\mathrm{MJD}=52357$ ). In general, the UB power spectra of GX $3+1$ are dominated by the VLFN with the brake at around $20 \mathrm{~Hz}$ at the lowest $k T_{e}=2.4 \mathrm{keV}$ (see blue B point on the right-hand panel of Figure 11 ). Specifically, during UB (blue histogram of panel (B) of Figure 11) one can see strong VLFN $\left(\mathrm{rms}=5.1 \% \pm 0.4 \%, \alpha_{\mathrm{LF}}=1.4 \pm 0.3, \chi^{2}=139\right.$ for 102 dof; all parameter errors correspond to $1 \sigma$ confidence level) and HFN with $\mathrm{rms}=1.7 \% \pm 0.3 \%$ and break frequency shifted from $30 \mathrm{~Hz}$ to $6 \mathrm{~Hz}$. After UB GX $3+1$ again returns to the LB showing similar to panel (A) properties of power density spectra (see panel (C) of Figure 11).

Note that these components and their CD evolution are typical for of atoll-sources in the banana state (Hasinger \& van der Klis 1989 ) and caused by mass accretion rate change (van der Klis 2005). This phase identification is supported by a combination of spectral (see Section 4.1) and timing properties in an agreement with atoll-Z scheme.

While the aforementioned $\mathrm{CD}$ evolution of power spectra of GX $3+1$ occurs on timescales from hours to days, we detected similar patterns for power spectrum evolution during LB-UB transitions for both faint phase and bright phase on luminosity during long-term variability within 14 years with a quasi-periodic trend during six years. The similarity of LB-UB transitions which are independent of bright/faint phases on luminosity indicates to similar accretion configurations in all phases. 


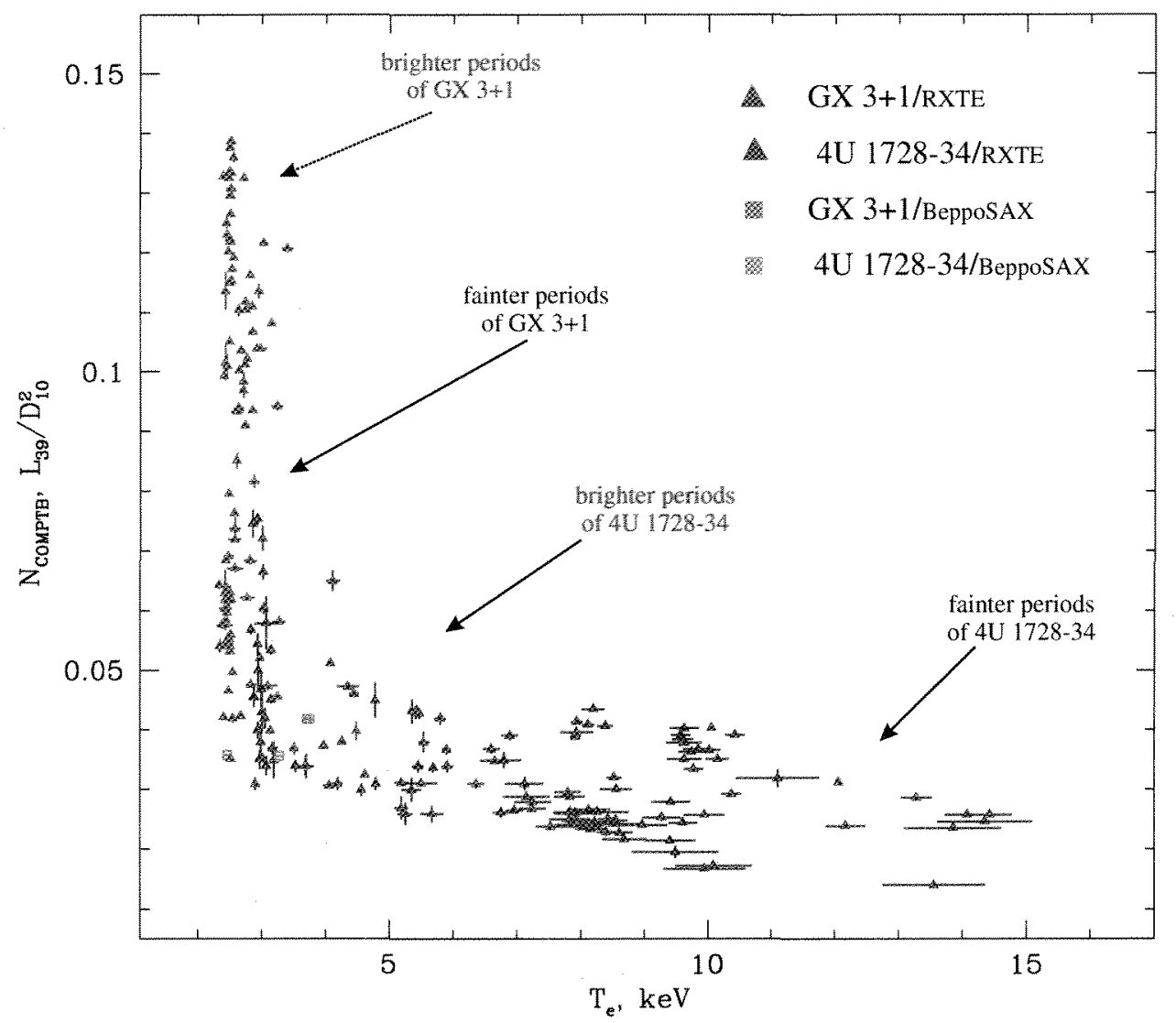

Figure 9. COMPTB normalization measured in $L_{39}^{\text {soft }} / D_{10}^{2}$ units vs. electron temperature $T_{e}$ (in keV) obtained using the best-fit spectral model wabs * (blackbody + COMPTB + Gaussian) for atoll sources GX $3+1$ (pink) and $4 \mathrm{U}$ 1728-34 (blue, taken from ST11) for RXTE data and bright blue and green points for BeppoSAX data. Mass accretion rate continuously increases along this correlation from the right to the left. (A color version of this figure is available in the online journal.)
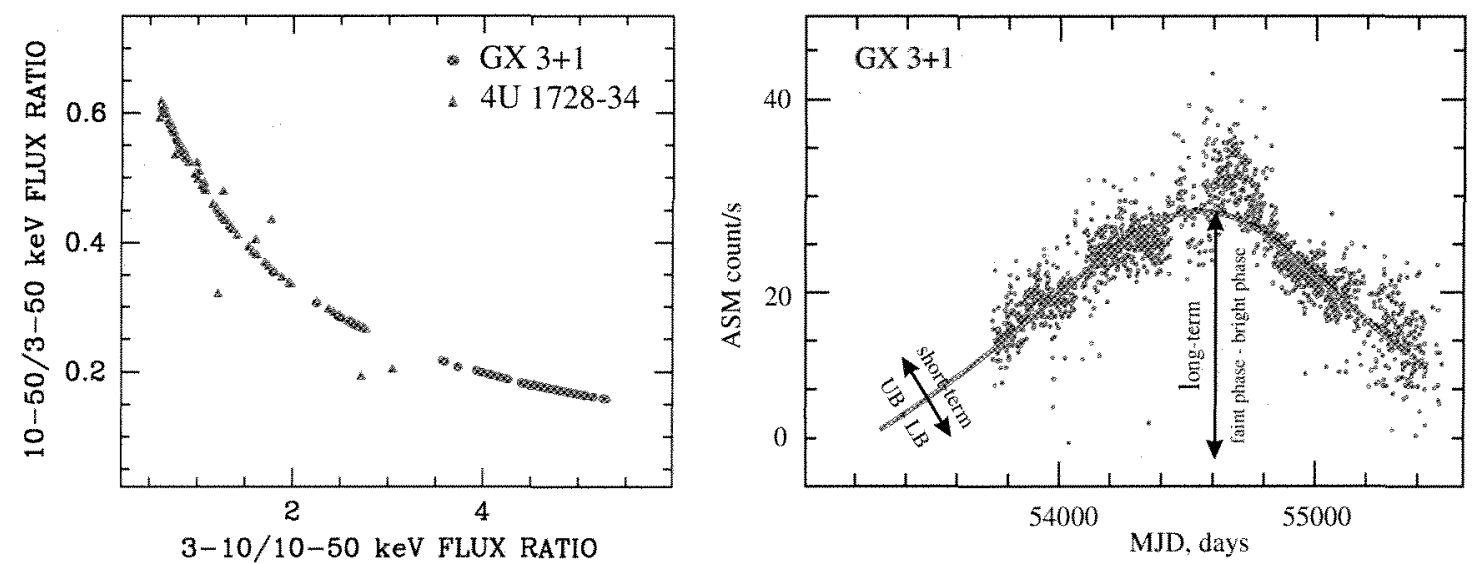

Figure 10. Left: the color-color diagram (3-10/10-50 keV flux ratio vs. 10-50/3-50 keV flux ratio) of GX $3+1$ (pink) and 4 U 1728-34 (blue) during faint-bright transitions (long-term variability). Right: fragment of ASM light curve of GX $3+1$ which shows two types of flux variability. One is a long-term trend (from faint to bright) related to COMPTB normalization changes and another one is short-term time variations (UB-LB) related to the electron temperature changes. The blue line presents a mean count rate and indicates to a long-term variability of $\mathrm{GX} 3+1$.

(A color version of this figure is available in the online jounal.)

In a previous analysis of other RXTE data of GX $3+1$ Oosterbroek et al. (2001) report VLFN and HFN values, in LB state, which are similar to our values in interval R3, with the exception of VLFN strength for which they report $7.5 \% \mathrm{rms}$ while we find $1.7 \%$ rms. All of the VLFN and HFN values of the analysis of EXOSAT data reported by Hasinger \& van der Klis (1989) for GX $3+1$ agree with our results.

\subsection{Comparison of Spectral and Timing Characteristics Atoll Sources $G X 3+1$ and $4 U 1728-34$}

\subsubsection{Quasi-constancy of the Photon Index}

The atoll sources $\mathrm{GX} 3+1$ and $4 \mathrm{U}$ 1728-34 demonstrate a similar behavior of the parameter $\Gamma$ versus mass accretion rate (or our COMPTB normalization), namely, the quasi-constancy 

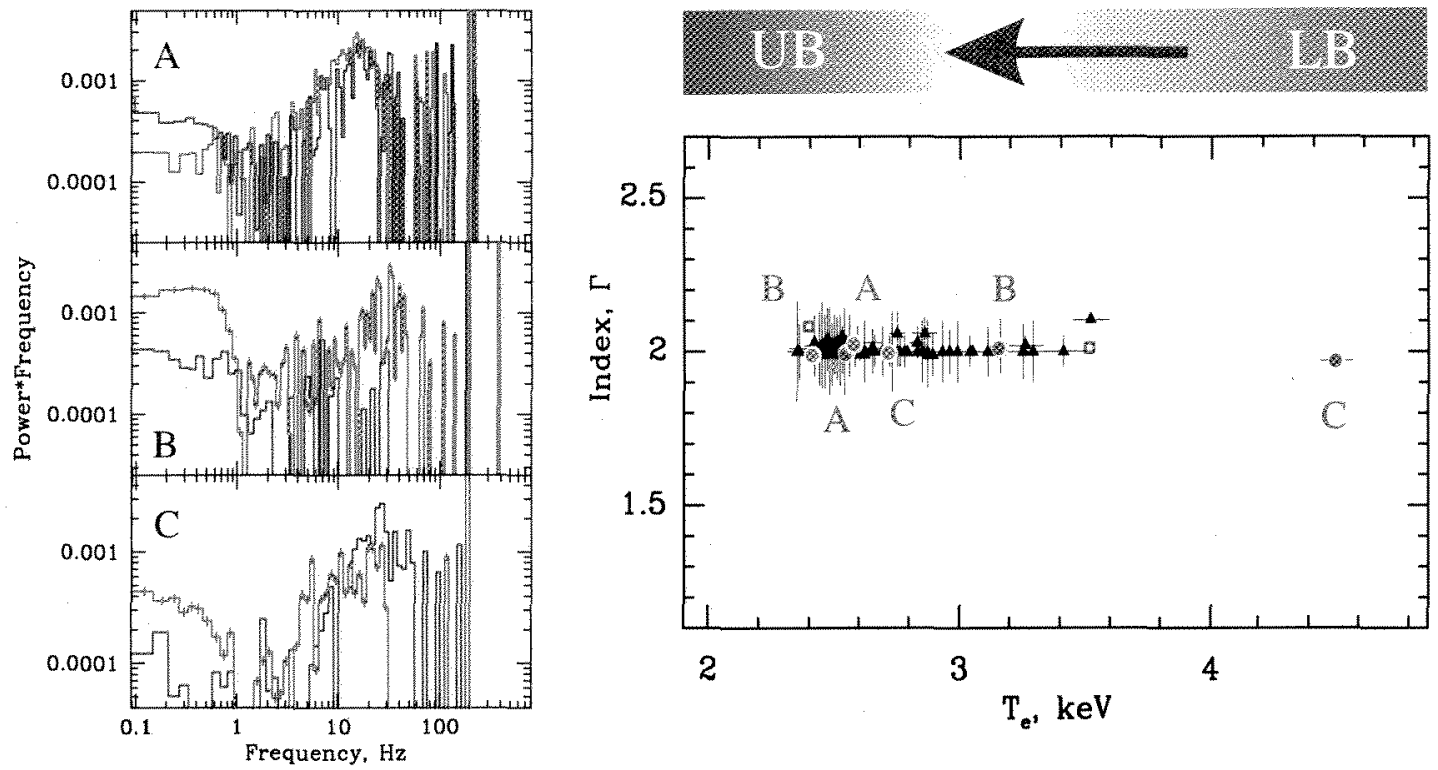

Figure 11. Left column: PDSs presented in terms of $v P(\nu)$ for $13-30 \mathrm{keV}$ energy band correspond to lower banana and upper banana states of GX $3+1$ and related to different electron temperatures indicated by points $\mathrm{A}, \mathrm{B}$, and $\mathrm{C}$ on the right panel. The strong noise component $\mathrm{HFN}$ seen in the $1-50 \mathrm{~Hz}$ range and relatively weak VLFN at the frequencies below $\sim 1 \mathrm{~Hz}$ are present before and after transition (see panels (A) and (C)) from LB to UB. In UB (panel (B), blue histogram) the power spectra of GX $3+1$ are dominated by the VLFN with a break at about $20 \mathrm{~Hz}$. Right panel: photon index $\Gamma$ plotted vs. electron temperature $T_{e}$ (in keV) using our spectral model wabs * (blackbody +COMPTB + Gaussian) during spectral transitions (see Table 4). Violet and black points correspond to BeppoSAX and $R X T E$ observations of $\mathrm{GX} 3+1$, respectively. The violet arrow in top part of the right panel points the direction of $L B \rightarrow \mathrm{UB}$ transition related to a decrease of $T_{e}$.

(A color version of this figure is available in the online joumal.)

Table 5

Comparisons of the Best-fit Parameters of Atoll Sources GX $3+1$ and 4 U 1728-341

\begin{tabular}{lcccccccc}
\hline \hline $\begin{array}{l}\text { Source } \\
\text { Name }\end{array}$ & $\begin{array}{c}\text { Alternative } \\
\text { Name }\end{array}$ & Class $^{2}$ & $\begin{array}{c}\text { Distance } \\
(\mathrm{kpc})\end{array}$ & $\begin{array}{c}\text { Presence of } \\
\mathrm{kHz} \text { QPO }\end{array}$ & $\begin{array}{c}k T_{e} \\
(\mathrm{keV})\end{array}$ & $\begin{array}{c}N_{\text {complb. }} \\
L_{39}^{\text {soft }} / D_{10}^{2}\end{array}$ & $\begin{array}{c}k T_{\mathrm{BB}} \\
(\mathrm{keV})\end{array}$ & $\begin{array}{c}k T_{s} \\
(\mathrm{keV})\end{array}$ \\
\hline 4U 1744-26 & GX 3+1 & Atoll, Sp, B & $4.5^{3}$ & none $^{5}$ & $2.3-4.5$ & $0.04-0.15$ & 0.6 & $1.16-1.7$ \\
$4 \mathrm{U} \mathrm{1728-34}$ & GX 354-0 & Atoll, Su, D & $4.2-6.4^{4}$ & $+^{6}$ & $2.5-15$ & $0.02-0.09$ & $0.2-0.9$ \\
\hline
\end{tabular}

References. (1) ST11; (2) Classification of the system in the various schemes (see the text): Sp: supercritical, Su: subcritical, B: bulge, D: disk; (3) Kuulkers \& van der Klis 2000; (4) van Paradijs 1978; (5) Strohmayer 1998; (6) Titarchuk \& Osherovich 1999.

of the photon index $\Gamma \approx 2$ and almost identical long-term variations of ASM mean count rate (see also ST11). According to FT11 and ST11 this observational fact can presumably indicate that the energy release in the TL for these two sources is much higher than cooling flux of the soft (disk) photons.

\subsubsection{A Difference of the Electron Temperature $k T_{e}$ Ranges in $G X 3+1$ and $4 U 1728-34$}

A comparison of the best-fit spectral parameters for these two atoll sources shows that a slow variability is generally related to changes of COMPTB normalization, and a mild variability is mainly correlated with the electron temperature variations (see Figure 10). Note that the ranges of disk and NS temperatures are similar for both of these objects, namely, $k T_{\mathrm{BB}} \simeq 0.6 \mathrm{keV}$ and $k T_{s}=1.2-1.7 \mathrm{keV}$, respectively. On the other hand variations of the electron temperature $k T_{e}$ are quite different. The electron temperature $k T_{\varepsilon}$ changes in a wide range $k T_{e}=2.5-15 \mathrm{keV}$ for $4 \mathrm{U} 1728-34$, while for GX $3+1 k T_{e}$ varies in a narrow range from 2.3 to $4.5 \mathrm{keV}$ (see Figures 8 and 9 ). The reason for this difference of temperature ranges is quite obvious. While $4 U$ 1728-34 shows an evolution from the extreme island state (EIS) to the UB state (see Di Salvo et al. 2001; ST11) GX 3+1 demonstrates only a short LB-UB track on CD (see Figure 8). These ranges of $\mathrm{CD}$ states are related to the ranges of $k T_{\ell}$.

\subsubsection{Comparison of Spectral Evolution as a Function of the COMPTB Normalization for $G X 3+1$ and $4 U 1728-34$}

We can also compare spectral parameter evolution for $\mathrm{GX} 3+1$ and $4 \mathrm{U}$ 1728-34 using COMPTB normalization because the distances to these sources are almost the same (see Table 5). Namely, for GX $3+1$ the distance is in the range of $4.2-6.4 \mathrm{kpc}$ (Kuulkers \& van der Klis 2000), whereas for $4 \mathrm{U} 1728-34$ it is $4.5 \mathrm{kpc}$ (van Paradijs 1978). In Figure 9, we show a correlation of COMPTB normalization presumably proportional to mass accretion rate and the electron temperature $k T_{e}$ for these two atoll sources. GX $3+1$ demonstrates a wider range of COMPTB normalization (by a factor of two higher than that for $4 U 1728$ 34) while $k T_{e}$ varies only from 2.5 to $4.5 \mathrm{keV}$. The common interval of $k T_{e}$ for $\mathrm{GX} 3+1$ and $4 \mathrm{U} 1728-34$ is in the range $2.5-4.5 \mathrm{keV}$ only and the low limit of the electron temperature of $2.5 \mathrm{keV}$ takes place at the peak luminosity for $4 \mathrm{U} 1728-34$ (see ST11) and during increases in luminosity for GX $3+1$, i.e., during the so-called UB state (see Figure 8 ).

\subsubsection{Correlation of Illumination Parameter fversus Electron Temperature $k T_{\ell}$ and Its Relation with Different States on Color-Color Diagram}

One can see from Table 5 that the range of illumination fraction of $\mathrm{CC}$ (TL) $f$ is wider for $\mathrm{GX} 3+1(0.1-0.9)$ than that 
for $4 U$ U 1728-34 (0.5-1). It can be related to different illumination of the TL for these two sources. For $4 U$ 1728-34 the solid angle viewed from NS changes by factor two whereas in GX $3+1$ that changes by factor four. However, the photon index $\Gamma \approx 2$ for these two sources which indicates that the energy release in the TL for these two sources is much higher than cooling flux of the disk photons (see FT11 and ST11 for details of X-ray spectral formation in TL).

In Figure 8, we present a plot $k T_{e}$ versus $f=A /(1+$ A) for atoll sources $\mathrm{GX} 3+1$ and $4 \mathrm{U}$ 1728-34 during mild variability. Pink/bright blue and blue/green points correspond to RXTE/BeppoSAX observations of GX $3+1$ and $4 \mathrm{U} 1728-34$, respectively. COMPTB normalization measured in $L_{39}^{\text {soft }} / D_{10}^{2}$ units versus $f$ is plotted in the incorporated top left panel for long-term (slow) variability of GX $3+1$ (see Table 4 for details). The bended arrows along the corresponding tracks correspond to an increase in mass accretion rate. On the right-hand side of figure we also present the sequence of $C D$ states which are listed according to the standard atoll- $\mathrm{Z}$ scheme (Hasinger \& van der Klis 1989). Here we also show that the CD sequence is definitely related to the electron temperature $k T_{e}$. The diagram of $T_{e}$ versus $f$ demonstrates a clear correlation $T_{e}$ and $f$ while the diagram $N_{\text {COM }}$ versus $f$, presented in the incorporated panel of Figure 8 , shows only chaotic scattering of points in a wide range of $f \sim 0.2-0.9$.

Moreover, we find two different track shapes on diagram of $T_{e}$ versus $f$ for two atoll sources $G X 3+1$ and $4 U$ 1728-34 and their relations with the standard sequence of $\mathrm{CD}$ states (Figure 8). When the fraction $f$ increases, the electron temperature $T_{e}$ decreases monotonically from approximately $4.5 \mathrm{keV}$ to $\sim 2.3 \mathrm{keV}$ for GX 3+1, while 4U 1728-34 demonstrates more complicated behavior pattern. At EIS, with a decrease of $T_{e}$, the fraction $f$ slightly varies from 0.9 to 1 . When $T_{e}$ further decreases from $12 \mathrm{keV}$ to $4 \mathrm{keV}, f$ decreases from 0.9 to 0.5 . Finally, during the LB-UB state transition $f$ increases from 0.5 to 1 .

Thus we show that the evolution CD states can be traced by the correlation between $T_{e}$ and $f$ too. Note that the tracks of $f-T_{e}$ on this diagram resemble the atoll tracks on the standard CD (Schultz et al. 1989).

\section{CONCLUSIONS}

We present our analysis of the spectral properties observed in X-rays from the NS X-ray binary GX $3+1$ during long-term transitions between the faint phase and the bright phase superimposed by short-term transitions between $L B$ and $U B$ states. We analyze all transition episodes for this source observed with BeppoSAX and RXTE. For our analysis we apply the good spectral coverage and resolution of BeppoSAX detectors from 0.1 to $200 \mathrm{keV}$ along with extensive RXTE coverage in the energy range from 3 to $50 \mathrm{keV}$.

We show that the $\mathrm{X}$-ray broadband energy spectra during all spectral states can be adequately fitted by the combination of a Blackbody, a Comptonized, and a Gaussian components. We also show that photon index $\Gamma$ of the best-fit Comptonized component in $G \times 3+1$ is almost constant, with a value of two and consequently is almost independent of COMPTB normalization $L_{39}^{\text {soft }} / D_{10}^{2}$ which is presumably proportional to mass accretion rate $\dot{m}$ (see Figures 6,7 , and 11 ). We should remind a reader that this index stability has recently been suggested using $X$-ray observations of quite a few of other NS sources. Namely atoll sources: X 1658-298, GS 1826-238, 1E 1724-3045, and also Z-sources: Cyg X-2, Sco X-1, GX 17+2, GX 340+0, GX 349+2 were observed by BeppoSAX at different spectral states and also atoll source 4 U 1728-34 observed by BeppoSAX and RXTE (see details in FT11 and ST11, respectively).

A wide variation of parameter $f=0.1-0.9$, obtained in the framework of our spectral model, points out a significant variation of the illumination of Comptonization region (TL) by $\mathrm{X}$-ray soft photons in GX $3+1$.

Using BeppoSAX observations we find that there are two sources of blackbody emission, one is presumably related to the accretion disk and another one is related to the NS surface for which temperatures of soft photons are about $0.7 \mathrm{keV}$ and $1.3 \mathrm{keV}$, respectively.

We demonstrate that our analysis of X-ray spectral and timing properties in atoll source GX $3+1$ allows us to distinguish between mild and long-term variabilities, and link them with LB-UB state transitions and transitions between bright and faint phases in luminosity, respectively. In this way we described mild flux variability between LB and UB states on timescale of hours-days in terms of two basic spectral parameters, the electron temperature $k T_{e}$ and illumination fraction $f$. We argue that $k T_{e}$ monotonically increases from $2.3 \mathrm{keV}$ to $4.5 \mathrm{keV}$ when GX $3+1$ makes a transition from UB state to LB state. We also find two noise components (VLFN and HFN) and their evolution during LB-UB transitions: the $X$-ray power spectra (PDS) in UB are dominated by VLFN with the break around $20 \mathrm{~Hz}$, whereas in LB the PDSs are dominated by an HFN in $1-100 \mathrm{~Hz}$ range and accompanied by reduced VLFN below $\sim 1 \mathrm{~Hz}$.

We demonstrate that the photon index $\Gamma \sim 2$ is approximately constant when the source moves from the faint phase to the bright phase and as well as during local transitions from $L B$ to UB. In ST11 we presented strong theoretical arguments that the dominance of the energy release in the TL with respect to the soft flux coming from the accretion disk, $Q_{\text {disk }} / Q_{\text {cor }} \ll 1$, leads to almost constant photon index $\Gamma \approx 2$.

Thus we argue that the stability of this index is an intrinsic signature of atoll sources while in $\mathrm{BH}$ s the index monotonically changes with mass accretion rate and ultimately saturates (see ST09 and ST11). Photon indices of BH candidates (GRS 1915+105, GX 339-4, SS 433, H1743-322, 4U 1543-47, Cyg X-1, XTE J1550-564, and GRO J1655-40) show clear correlation with mass accretion rate $\dot{m}$ (ST09, TS09, and Seifina \& Titarchuk 2010). This correlation is accompanied by an index saturation when $\dot{m}$ exceeds a certain level. The behaviors of the index for $\mathrm{GX} 3+1$ and $4 \mathrm{U} \quad 1728-34$ are clearly different from that for the sample of BHC sources. The photon index $\Gamma \approx 2$ while mass accretion rate changes by factor four.

We acknowledge discussion and editing of the paper content with Chris Shrader. We are very grateful to the referee whose constructive suggestions help us to improve the paper quality.

\section{REFERENCES}

Asai, K., Dotani, T., Nagase, F., et al. 1993, PASJ, 45,80 ?

Boella, G., Chiappett, L., Cont, G., et al. 1997, A.\&AS, 122, 327 Bowyer, S., Byram, E. T., Chubb, T. A., \& Fredman, H. 1965, Science, 147, 394

Bradt, H. V., Rothschid, R. E., \& Swank, J. H. 1993, A\&AS, 97, 355

Chenevez, J., Falanga, M., Brandt, S., et al, 2006, ABA, 449, $S_{5}$

Christian, D. J., \& Swank, J. H. 1997, Anw, 109, 177

Cumming, A., \& Bildsten, L. 2001, Aws, 559, L 127

den Hartog, P. R., in't Zand, J. J. M., Kunkers, E., et al. 2003, A \& A, 400, 663

Di Salvo, T., Mendez, M., van der Klis, M., Ford, E., \& Robba, N. R. 2001, Ap, 546. 1107

Farinell, R., \& Thtarchuk, L. 2011, A\&A, 525, 102 (FTl1)

Farinelli, R., Titarchuk, L., Paizis, A., \& Frontera, F. 2008, Ap, 680,602 (F08) Ford, E. C., van der Klis, M., Mendez, M., et al. 2000, Apl, 537,368 
Frontera, F., Costa, E., dal Fiume, D., et al 1997, Proc. SPIE, 3114, 206

Galloway, D. K., Muno, M. P., Hartman, J. M., et al. 2008, Av1 \$, 179, 360

Hasinger, G., \& van der Klis, M. 1989, A\&A, 225,79

Homan, J., van der Klis, M., Wijnands, R., Vaughan, B., \& Kuulkers, E. 1998, A $\mathrm{Al}, 499,1 \mathrm{Al}$

Hanson, C.J., \& van Hom, H. M. 1975, Aps, 195, 735

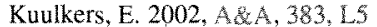

Kuulkers, E., \& van der Klis, M. 2000, A\&A, 356, 145

Lewin, W. H. G., van Paradijs, J., Hasinger, G., et al. 1987, MNRAS, 226, 383

Makishima, K., Mitsuda, K., Inoue, H., et al. 1983, Av, 267, 310

Molkov, S. V., Grebenev, S. A., Pavlinskij, M. N., \& Sunyaev, R. A. 1999, in

Proc. 3rd INTEGRAL Workshop on The Extreme Universe, ApLc, 38, 141

Naylor, T., Charles, P. A., \& Longmore, A. J. 1991, MNRAS, 252,203

Oosterbroek, T., Barret, D., Gianazzi, M., \& Ford, E. C. 2001, A\& A, 366. 138

Paizis, A., Farinelli, R., Titarchuk, L., et al. 2006, A\&A, 459. 187

Parmar, A. N., Martin, D. D. E., Bavdaz, M., et al. 1997, A\& A5, 122,309

Pavlinsky, M. N., Grebenev, S. A., \& Sunyaev, R. A. 1994, Apl, 425. 110

Schnerr, R. S., Reerink, T., van der Klis, M., et al. 2003, A.

Schultz, N. S., Hasinger, G., \& Trumper, J. 1989, A\&A, 225, 48

Seifina, E., \& Titarchuk, L. 2010, Ap), 722,586 (ST10)

Seifina, E., \& Titarchuk, L. 2011, ApJ, 737, 128 (ST11)

Shakura, N. I., \& Sunyaev, R. A. 1973, A\&A, 24, 337

Shaposhnikov, N., \& Titarchuk, L. 2009, Apj, 699, 443 (ST09)

Stella, L., White, N. E., \& Taylor, B. G. 1985, in Recent Results on Cataclysmic Variables, 125

Strohmayer, T. 1998, in AP Conf. Sew, 397 (arXiv:astro-phy802022v1)

Strohmayer, T. E., \& Brown, F. B. 2002, Apl, 566,1045

Titarchuk, L., \& Shaposhnikov, N. 2010, Avs, 724, 1147

Titarchuk, L., \& Seifina, E. 2009, An, 706, 1463

Titarchuk, L. G., \& Osherovich, V. A. 1999, Ap, $518,2,95$

van der Klis, M. 2005, ApdSS, 300, 140

van der Klis, M. 2000, ARA\&, 38,717

van Paradijs, J. 1978, Nawre, 274, 650

Woosley, S. E., \& Taam, R. E. 1976, Nature, 263.101

Wijnands, R., van der Klis, M., \& van Paradijs, J. 1998, in IAU Symp. 188, The Hot Universe, ed. K. Koyama, S. Kitamoto, \& M. Itoh (Dordrecht: Kluwer), 370 


\section{Queries}

\section{Page 1}

Q1

Author: Please provide department name in affiliation addresses 1 and 3.

Q2

Author: In reference citation "Homan et al. (1998)" the year has been changed from " 1975 " to " 1998 " here and elsewhere in the text to match the reference list. Please confirm if it is correct.

\section{Page 3}

Q3

Author: The same acronym "PDS" has been used for "Phoswich Detection System" and "power density spectra." Please check.

\section{Page 5}

Q4 Author: Please provide the corresponding text for footnote " $b$ " in Table 3.

\section{Page 7}

Q5

Author: Please confirm whether the edit made to the sentence "Thus, in order to fit the..." retains the intended sense.

Page 14

Q6

Author: Please check the details for any journal references that do not have a pale purple link (CrossRef doi) or a blue link (NASA ADS or arXiv e-print) in the two-column proof (articlestyle layout). A journal reference with no links may contain some incorrect information.

Q7

Author: Please cite references "Cumming \& Bildsten (2001)" and "Strohmayer \& Brown (2002)" in the text or else delete them from the reference list.

\section{Page 15}

Q8

Author: Please confirm whether the initials of the first author are correct as amended in reference "Galloway et al. (2008)."

Q9

Author: Please provide editors' names and publisher details (name and location) in reference "Molkov et al. (1999)" and "Stella et al. (1985)."

Q10

Author: Please provide conference title, editors' names, publisher details (name and location), and page number in reference "Strohmayer (1998)."

Q11

Author: Please confirm whether editors' names and publisher details (name and location) are correct as included in reference "Wijnands et al. (1998)."

\section{Online-only colour figures}

This proof PDF is identical in specification to the PDF file that will be published in the online journal. To view any online-only color figures as they will appear in the printed journal, we recommend that this color PDF file be printed on a black \& white printer. 